\title{
COVID-19, MERS and SARS with Concomitant Liver Injury-Systematic Review of the Existing Literature
}

\author{
Michał Kukla ${ }^{1,2,3,+} \mathbb{0}$, Karolina Skonieczna-Żydecka ${ }^{4,+}{ }^{\circledR}$, Katarzyna Kotfis ${ }^{5}{ }^{\circledR}$, \\ Dominika Maciejewska ${ }^{4}$ (D), Igor Łoniewski ${ }^{4}$, Luis. F. Lara ${ }^{6}$, Monika Pazgan-Simon ${ }^{3,7}$, \\ Ewa Stachowska ${ }^{4}$ (D), Mariusz Kaczmarczyk ${ }^{8}$, Anastasios Koulaouzidis ${ }^{9}$ (D) and \\ Wojciech Marlicz 10,*(D)
}

1 Department of Internal Medicine and Geriatrics, Jagiellonian University Medical College, 2 Jakubowskiego St., 30-688 Cracow, Poland; kuklamich@poczta.onet.pl

2 Department of Endoscopy, University Hospital in Cracow, 2 Jakubowskiego St., 30-688 Cracow, Poland

3 1st Infectious Diseases Ward, Gromkowski Regional Specialist Hospital, Wroclaw, 5 Koszarowa St., 50-149 Wroclaw, Poland; monika.pazgan.simon@gmail.com

4 Department of Human Nutrition and Metabolomics, Pomeranian Medical University in Szczecin, 71-460 Szczecin, Poland; karzyd@pum.edu.pl (K.S.-Ż.); dmaciejewska.pum@gmail.com (D.M.); sanprobi@sanprobi.pl (I.Ł.); ewa.stachowska@pum.edu.pl (E.S.)

5 Department of Anaesthesiology, Intensive Therapy and Acute Intoxications, Pomeranian Medical University in Szczecin, 70-111 Szczecin, Poland; katarzyna.kotfis@pum.edu.pl

6 Division of Gastroenterology, Hepatology, and Nutrition, The Ohio State University Wexner Medical Center, Columbus, OH 43210, USA; Luis.Lara@osumc.edu

7 Department of Infectious Diseases, Wroclaw Medical University, 5 Koszarowa St., 50-149 Wroclaw, Poland

8 Department of Clinical and Molecular Biochemistry, Pomeranian Medical University in Szczecin, 70-111 Szczecin, Poland; mariush@pum.edu.pl

9 Centre for Liver \& Digestive Disorders, Royal Infirmary of Edinburgh, Edinburgh EH16 4SA, UK; akoulaouzidis@hotmail.com

10 Department of Gastroenterology, Pomeranian Medical University, 71-252 Szczecin, Poland

* Correspondence: marlicz@hotmail.com; Tel.: +48-91-425-3231

+ These authors contributed equally to this work.

Received: 10 April 2020; Accepted: 6 May 2020; Published: 11 May 2020

\begin{abstract}
The novel coronavirus SARS-CoV-2 (severe acute respiratory syndrome coronavirus 2) infection has been predominantly linked to respiratory distress syndrome, but gastrointestinal symptoms and hepatic injury have also been reported. The mechanism of liver injury is poorly understood and may result as a consequence of viral hepatitis, systemic inflammatory response, gut barrier and microbiome alterations, intensive care treatment or drug toxicity. The incidence of hepatopathy among patients with coronavirus disease 2019 (COVID-19) is unclear, but studies have reported liver injury in patients with SARS and Middle East respiratory syndrome (MERS). We aimed to systematically review data on the prevalence of hepatic impairments and their clinical course in SARS and MERS Coronaviridae infections. A systematic literature search (PubMed/Embase/Cinahl/Web of Science) according to preferred reporting items for systematic review and meta-analysis protocols (PRISMA) was conducted from database inception until 17/03/2020 for studies that evaluated the incidence of hepatic abnormalities in SARS CoV-1, SARS CoV-2 and MERS infected patients with reported liver-related parameters. A total of forty-three studies were included. Liver anomalies were predominantly mild to moderately elevated transaminases, hypoalbuminemia and prolongation of prothrombin time. Histopathology varied between non-specific inflammation, mild steatosis, congestion and massive necrosis. More studies to elucidate the mechanism and importance of liver injury on the clinical course and prognosis in patients with novel SARS-CoV-2 infection are warranted.
\end{abstract}


Keywords: SARS-CoV-2; coronavirus; liver; pandemic; SARS; MERS; COVID-19

\section{Introduction}

The novel coronavirus named SARS-CoV-2 (severe acute respiratory syndrome coronavirus 2) was declared a public health emergency of international concern on 30 January, 2020 after the outbreak of severe pulmonary infections of unknown cause that started in Wuhan, Hubei province, China, in December 2019 [1,2]. The term coronavirus disease 2019 (COVID-19) was designated by the World Health Organization (WHO) on the 11th of February 2020 to be used for the severe pneumonia associated with SARS-CoV-2 infection. On Wednesday, 11 March 2020, COVID-19 was declared a pandemic by the WHO, as it affected more countries and threatened the lives of patients worldwide [3]. Several groups of investigators have already reported frequent gastrointestinal symptoms in COVID-19 patients which may be associated with increased disease severity [4,5], including prolonged coagulation times and elevated liver serum tests compared to those without digestive symptoms $[6,7]$. Hepatopathy has been associated with disease severity with two other highly pathogenic coronavirus strains-severe acute respiratory syndrome coronavirus 1 (SARS-CoV-1) and the Middle East respiratory syndrome coronavirus (MERS-CoV) [8]. Recent clinical studies of COVID-19 indicate that hepatic injury presents with elevated transaminases, elevated bilirubin, prolonged prothrombin time and hypoproteinemia [9] and the severity of blood test anomalies may predict a poorer outcome. Bangash et al. reported that mild laboratory anomalies were not prognostic of outcomes [10]; however, $58 \%$ to $78 \%$ of patients with severe COVID-19 associated disease presented with varying degrees of liver injury [11], therefore clinically significant liver dysfunction may be an important feature of and may predict the prognosis of COVID-19 [2,9,12]. The pathogenesis is likely multifactorial including: (i) viral hepatitis (hepatic viral replication); (ii) hypoxic hepatitis (secondary to respiratory failure); (iii) hepatic congestion associated with mechanical ventilation (high levels of positive end-expiratory pressure (PEEP)); (iv) drug toxicity (antiviral medications, anti-malaria medications, antibiotics, steroids); (v) immune response (virally induced intrahepatic cytotoxic $\mathrm{T}$ cells and Kupffer cells) and (vi) gut vascular barrier and microbiota alterations [10]. In the most severe cases of COVID-19 hepatic dysfunction has been associated with an activation of coagulation and fibrinolysis accompanied by thrombocytopenia [12].

Previous studies regarding SARS and MERS indicated that approximately $60 \%$ of patients presented with hepatopathy, mostly in the form of elevated liver enzymes [13]. Severe COVID-19 disease has been associated with a high prevalence of abnormal aminotransferase levels; however, they may also be of non-hepatic origin [12]. We aimed to systematically review the existing data to determine the correlation of hepatic involvement in patients with either SARS-CoV-1, MERS-CoV or SARS-CoV-2 infection, and determine a relationship with outcomes, especially prognosis in patients with COVID-19.

\section{Materials and Methods}

\subsection{Search Strategy and Selection Criteria}

The preferred reporting items for systematic review and meta-analysis protocols (PRISMA) methodology [14] was used as the search strategy. Two blinded authors (K.S.-Ż. and D.M.) performed a systematic search in the following databases: PubMed/Medline/Embase/Cinahl/Web of Science from database inception until 17 March 2020. The search string utilized was: (("coronaviridae" (Terms) OR "coronaviridae" (All Fields)) OR ("coronavirus infections" (MeSH Terms) OR ("coronavirus" (All Fields) AND “infections"(All Fields)) OR "coronavirus infections" (All Fields) OR ("coronavirus" (All Fields) AND "infection" (All Fields)) OR "coronavirus infection" (All Fields))) AND ("liver" (MeSH Terms) OR “liver" (All Fields)) AND ("humans" (MeSH Terms) OR “humans" (All Fields) OR 
"human" (All Fields)). We did not include reviews, meta-analyses and systematic reviews; however, any commentary, editorial, letter to editors, abstract where clinical data were provided was included for further review and data extraction. After screening of the aforementioned databases, the search was supplemented by manual review of references covering the topic of interest.

\subsection{Inclusion Criteria}

- Human retrospective studies, including case reports and case series reporting clinical liver-related data in patients infected with SARC-CoV-1, SARS-CoV-2 and MERS;

- Any of the following clinical data: alanine aminotransferase (ALT)/aspartate aminotransferase (AST)/gamma-glutamyl-transpeptidase (GGTP)/alkaline phosphatase (ALP)/lactate dehydrogenase (LDH)/creatinine (Cr)/bilirubin (BIL)/total protein (TP)/albumin (ALB)/international normalised ratio (INR)/prothrombin time expressed either qualitatively (percent of abnormal results) or quantitatively (PT);

- Post-mortem studies reporting liver histopathology.

Pediatric patients were excluded; however, when a study reported data on both children and adults the data were evaluated separately for persons above the age of 18 years. In addition, for studies originating in China we extracted data only for abstracts in English.

\subsection{Data Extraction and Analysis}

At least two authors (K.S.-Ż., D.M. and M.K. (Michał Kukla)) independently abstracted data on basic study characteristics (type of coronavirus, study aim, type of study, i.e., in vivo/post mortem), study subjects (age, comorbidities), intervention (drugs, intensive care treatment) and liver related parameters. When abstracting data from figures, WebPlot digitizer software was used (https:// automeris.io/WebPlotDigitizer/). The data were stored in an Excel file by each investigator and after the comparison of the file content all discrepancies were solved by the third author by consensus. The significance of the analyzed studies was arbitrarily assigned as follows: strong-full text studies with data on more than 30 patients, middle—studies comprising a small number of patients $(<30)$, weak-case reports and abstracts with no access to full text.

\subsection{Risk of Bias Assessment}

Two authors (K.S.-Ż. and D.M.) independently assessed the risk of bias using the Strengthening the Reporting of OBservational Studies in Epidemiology (STROBE) assessment [15]. However, item 16 (unadjusted estimates, confounder-adjusted estimates, category boundaries, translating estimates of relative risk into absolute risk for a meaningful period), was omitted as it was not applicable to the present study. Each item was evaluated, and 1 point was added for each when fulfilled. Therefore, the rating was the sum of points received for each STROBE item. The highest number of points obtainable in each study was 31 .

\section{Results}

\subsection{Descriptive Data}

The initial search yielded 279 results, of which 222 were excluded after reviewing titles and abstracts. Fifty-seven manuscripts were fully reviewed, plus 9 more studies which were manually found. Subsequently 23 studies were excluded due to lack of liver-related clinical data $(n=10)$; pediatric patients $(n=5)$; no access to English abstract $(n=3)$; no access to full texts $(n=2)$; and one each $(n=1)$ including animal study, duplicate and incomplete abstract. Thus, 43 articles comprised the study group $[2,4,8,12,13,16-53]$ (Figure 1). 
Most of the studies $(n=35)[2,4,12,13,16-25,27,30-33,35-38,40-42,44-52]$ were observational and retrospective. We extracted data from eleven $(n=11)$ English abstracts provided for Chinese articles $[17,26,30,32,34,35,37,38,42,44,48]$ and for one $(n=1)$ commentary [19].

There were eight $(n=8)$ post-mortem studies ( 7 full texts, 1 abstract) $[8,26,28,29,34,39,43,53]$. There were $11(n=11)[2,4,12,16-23]$ studies focusing on SARS CoV-2 and $23(n=23)[13,24-45]$ which reported data on patients infected with SARS CoV-1. MERS had the last amount of data $(n=9)$ [8,46-53]. Overall, we analyzed clinical characteristics of 4591 subjects, most with SARS CoV-2 $(n=2541)$, followed by SARS CoV-1 $(n=1894)$ and MERS $(n=156)$. The mean/median age, when provided, was between 33 and 45.21 years. Comorbidities were reported in 14 studies including hypertension (HTN) in 306, diabetes mellitus (DM) in 171 and cardiovascular diseases (CVD) in 112 patients. Obesity status $(n=7)$ and body mass index (BMI) value $\left(30.5 \mathrm{~kg} / \mathrm{m}^{2}\right)$ were provided in a single study each. Other comorbidities included asthma, chronic obstructive pulmonary disease (COPD), kidney diseases and others, with a total number of 398 cases. Treatment data were provided in 24 studies; however, drugs dosages and duration of intervention were predominantly missing. Mechanical ventilation was reported in 407 patients in 24 studies. The reported study, patient and treatment characteristics for all three types of coronaviruses are provided in Table 1.

\subsection{Liver-Related Outcomes}

The most frequently reported data were concentrations of ALT $(n=19)$ and AST $(n=16)$, followed by bilirubin $(n=14)$ and serum creatinine $(n=12)$. Raw data on albumin content, prothrombin time, LDH and ALP were reported by 10, 8, 6 and 4 studies, respectively. Quantitative data including number and percentage of patients with abnormal tests were extracted. The most commonly reported were ALT $(n=14)$, AST $(n=9)$ and creatinine $(n=6)$. Normal ranges varied by study (ALT: $>40-53 \mathrm{U} / \mathrm{L}$;

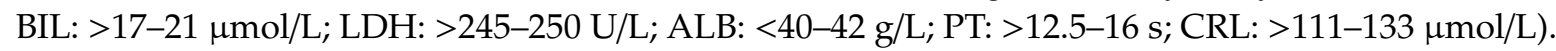
The data are presented in Table 2. Twelve studies reported liver histopathology, seven $(n=7)$ were autopsy studies. The details are provided in Table 3.

\subsection{Risk of Bias (ROB)}

The ROB evaluation was performed using the STROBE assessment tool in 28 full text manuscripts. None of the studies reached 31 points. None of the studies described methods to address risk of bias within their observations. The biggest variations occurred regarding the study size and statistical methods, in particular, the quantitative variables data. The mean number of points achieved was $26.54 \pm 2.5$ points. The lowest number of points achieved was 23 , and the highest was 30 . The detailed scoring is reported in Supplementary Table S1. 
Table 1. Study and patient characteristics.

\begin{tabular}{|c|c|c|c|c|c|c|c|c|}
\hline $\begin{array}{l}\text { Reference/Year/ } \\
\text { Country }\end{array}$ & $\begin{array}{l}n \text { Total/ } \\
n \text { Males }\end{array}$ & $\begin{array}{c}\text { Age } \\
(\text { Mean/SD) }\end{array}$ & $\begin{array}{l}\text { Mechanical } \\
\text { Ventilation/ } \\
\text { Suppl Oxygen }\end{array}$ & $\begin{array}{l}\text { Hospitalization/ } \\
\text { ICU Stay }\end{array}$ & $\begin{array}{l}\text { Hospital } \\
\text { Mortality/ICU } \\
\text { Mortality }\end{array}$ & $\begin{array}{l}\text { Comorbidities } \\
\text { (Diabetes/ } \\
\text { Hypertension/ } \\
\text { CVD/Other) }\end{array}$ & $\begin{array}{l}\text { Preexisting } \\
\text { Liver Disease } \\
\text { (n)/Diagnosis }\end{array}$ & Intervention \\
\hline \multicolumn{9}{|c|}{ SARS CoV-2 } \\
\hline $\begin{array}{l}\text { Chen et al. [16]/ } \\
\text { 2020/China }\end{array}$ & $99 / 67$ & $55.5 / 13.1$ & $17 / 75$ & 99/nd & $31 / \mathrm{nd}$ & $\mathrm{nd} / \mathrm{nd} / \mathrm{nd} / 0$ & nd/nd & \multirow{5}{*}{$\begin{array}{c}\text { antibiotic, antiviral treatment } \\
\text { nd } \\
\text { antibiotics }(n=637) \text {, oseltamivir } \\
(n=393) \text {, antifungals }(n=31) \text {, systemic } \\
\text { glucocorticoids }(n=204) \\
\text { moxifloxacin }(n=89), \text { ceftriaxone } \\
(n=34), \text { azithromycin }(n=25), \\
\text { glucocorticoids }(n=62) \\
\text { antibiotics }(n=181) \text {, antivirals }(n=41), \\
\text { corticosteroids }(n=57), \\
\text { immunoglobulins }(n=46)\end{array}$} \\
\hline $\begin{array}{l}\text { Chuan et al. [17]/ } \\
\text { 2020/China }\end{array}$ & $32 / \mathrm{nd}$ & nd/nd & nd/nd & $32 / \mathrm{nd}$ & $\mathrm{nd} / \mathrm{nd}$ & $\mathrm{nd} / \mathrm{nd} / \mathrm{nd} / 5$ & $\mathrm{nd} / \mathrm{nd}$ & \\
\hline $\begin{array}{l}\text { Guan et al. [2]/ } \\
\text { 2020/China }\end{array}$ & $1099 / 640$ & $47 / 35.58$ & $81 / 454$ & $1029 / 55$ & $15 /$ nd & $81 / 165 / 27 / 261$ & 23/HBV & \\
\hline $\begin{array}{l}\text { Wang et al. [12]/ } \\
\text { 2020/China }\end{array}$ & $138 / 75$ & $56 / 26$ & $32 / 106$ & $138 / 36$ & $6 / \mathrm{nd}$ & $14 / 43 / 20 / 0$ & $\begin{array}{l}\text { 4/chronic liver } \\
\text { disease }\end{array}$ & \\
\hline $\begin{array}{l}\text { Zhou et al. [18]/ } \\
\text { 2020/China }\end{array}$ & $191 /$ nd & $\mathrm{nd} / \mathrm{nd}$ & $58 / 41$ & $191 / 50$ & $54 / \mathrm{nd}$ & $36 / 58 / 15 / 22$ & $\mathrm{nd} / \mathrm{nd}$ & \\
\hline $\begin{array}{l}\text { Zhang et al. [19]/ } \\
\text { 2020/China }\end{array}$ & $56 /$ nd & $\mathrm{nd} / \mathrm{nd}$ & nd/nd & $56 /$ nd & nd/nd & $\mathrm{nd} / \mathrm{nd} / \mathrm{nd} / 0$ & $2 / \mathrm{nd}$ & \multirow{4}{*}{$\begin{array}{l}\text { nd } \\
\text { vasoconstrictive agents }(n=18), \\
\text { antivirals }(n=23), \text { antibacterials } \\
(n=49), \text { glucocorticoids }(n=30), \\
\text { immunoglobulin }(n=28) \\
\text { antivirals }(n=55), \text { antibiotics }(n=28), \\
\text { systematic corticosteroid }(n=16) \\
\text { antibiotic treatment }(n=73), \text { antivirals } \\
(n=80), \text { hormone therapy }(n=12), \\
\text { immunoglobulins }(n=16)\end{array}$} \\
\hline $\begin{array}{l}\text { Yang et al. [20]/ } \\
\text { 2020/China }\end{array}$ & $52 / 35$ & $59.7 / 13.2$ & $37 / 33$ & $52 / 52$ & $\mathrm{nd} / 32$ & $9 / \mathrm{nd} / 7 / 34$ & nd/nd & \\
\hline $\begin{array}{l}\text { Xu et al. [21]/ } \\
\text { 2020/China }\end{array}$ & $62 / 35$ & $41 / 20$ & $1 / \mathrm{nd}$ & $61 / 1$ & $0 / 0$ & $1 / 5 / \mathrm{nd} / 3$ & $7 /$ nd & \\
\hline $\begin{array}{l}\text { Wu et al. [22]/ } \\
\text { 2020/China }\end{array}$ & $80 / 39$ & $46.1 / 15.42$ & $0 / 35$ & $80 /$ nd & $0 / 0$ & $\mathrm{nd} / \mathrm{nd} / 25 / 12$ & $1 / \mathrm{nd}$ & \\
\hline $\begin{array}{l}\text { Shi et al. [23]/ } \\
\text { 2020/China }\end{array}$ & $81 / 42$ & $49.5 / 11$ & nd/nd & $81 /$ nd & $3 / \mathrm{nd}$ & $10 / 12 / 8 / 0$ & $\begin{array}{l}\text { 7/liver cirrhosis, } \\
\text { hepatitis }\end{array}$ & nd \\
\hline $\begin{array}{l}\text { Jin et al. [4]/ } \\
\text { 2020/China }\end{array}$ & $651 / 331$ & $45.21 / 14.42$ & $17 /$ nd & $\mathrm{nd} / 17$ & $\mathrm{nd} / \mathrm{nd}$ & $48 / 100 / 5 / 8$ & $25 /$ nd & $\begin{array}{l}\text { antivirals }(n=546) \text {, antibiotics }(n=277), \\
\text { glucocorticoids }(n=74),\end{array}$ \\
\hline \multicolumn{9}{|c|}{ SARS CoV-1 } \\
\hline $\begin{array}{l}\text { Chan et al. [24]/ } \\
\text { 2004/China }\end{array}$ & $118 / 55$ & $33 * /(20-18)^{\#}$ & $16 /$ nd & $\mathrm{nd} / \mathrm{nd}$ & $9 /$ nd & $\mathrm{nd} / \mathrm{nd} / \mathrm{nd} / 16$ & $12 / \mathrm{HBV}$ & lamivudine \\
\hline $\begin{array}{l}\text { Chan et al. [25]/ } \\
\text { 2005/China }\end{array}$ & $294 / 126$ & $36 * /(12-83)^{\#}$ & $33 /$ nd & $194 / 141$ & $27 /$ nd & $5 / 12 / 6 / 18$ & $30 / \mathrm{HBV}$ & $\begin{array}{l}\text { cefotaxime, clarithromycin, } \\
\text { oseltamivircorticosteroids, ribavirin, } \\
\text { lamivudine }\end{array}$ \\
\hline
\end{tabular}


Table 1. Cont.

\begin{tabular}{|c|c|c|c|c|c|c|c|c|}
\hline $\begin{array}{l}\text { Reference/Year/ } \\
\text { Country }\end{array}$ & $\begin{array}{l}n \text { Total/ } \\
n \text { Males }\end{array}$ & $\begin{array}{c}\text { Age } \\
(\text { Mean/SD) }\end{array}$ & $\begin{array}{l}\text { Mechanical } \\
\text { Ventilation/ } \\
\text { Suppl Oxygen }\end{array}$ & $\begin{array}{l}\text { Hospitalization/ } \\
\text { ICU Stay }\end{array}$ & $\begin{array}{l}\text { Hospital } \\
\text { Mortality/ICU } \\
\text { Mortality }\end{array}$ & $\begin{array}{l}\text { Comorbidities } \\
\text { (Diabetes/ } \\
\text { Hypertension/ } \\
\text { CVD/Other) }\end{array}$ & $\begin{array}{l}\text { Preexisting } \\
\text { Liver Disease } \\
\text { (n)/Diagnosis }\end{array}$ & Intervention \\
\hline $\begin{array}{l}\text { Chau et al. [13]/ } \\
\text { 2004/China }\end{array}$ & $3 / 0$ & $34.7 / 8.2$ & nd/nd & $3 / \mathrm{nd}$ & $3 /$ nd & $\mathrm{nd} / \mathrm{nd} / \mathrm{nd} / \mathrm{nd}$ & $\mathrm{nd} / \mathrm{nd}$ & $\begin{array}{l}\text { ceftriaxone, clarithromycin, Kaletra, } \\
\text { methylprednisolone or levofloxacin } \\
\text { alone }\end{array}$ \\
\hline $\begin{array}{l}\text { Chen et al. [26]/ } \\
\text { 2003/China }\end{array}$ & $7 / \mathrm{nd}$ & nd/nd & nd/nd & nd/nd & nd/nd & nd/nd/nd/nd & $\mathrm{nd} / \mathrm{nd}$ & nd \\
\hline $\begin{array}{l}\text { Cui et al. [27]/ } \\
\text { 2004/China }\end{array}$ & $182 / 103$ & $\mathrm{nd} /(11-86)^{\#}$ & $\mathrm{nd} / \mathrm{nd}$ & 57/nd & $\mathrm{nd} / \mathrm{nd}$ & $\mathrm{nd} / \mathrm{nd} / \mathrm{nd} / \mathrm{nd}$ & $\mathrm{nd} / \mathrm{nd}$ & $\begin{array}{c}\text { antibiotics }(n=160), \text { ribavirin } \\
(n=137), \text { methylprednisolone }(n=115)\end{array}$ \\
\hline $\begin{array}{l}\text { Ding et al. [28]/ } \\
\text { 2003/China }\end{array}$ & $3 / 2$ & $48 / 16.4$ & nd/nd & $\mathrm{nd} / \mathrm{nd}$ & $\mathrm{nd} / \mathrm{nd}$ & $\mathrm{nd} / \mathrm{nd} / \mathrm{nd} / \mathrm{nd}$ & $\mathrm{nd} / \mathrm{nd}$ & nd \\
\hline $\begin{array}{l}\text { Farcas et al. [29]/ } \\
\text { 2005/Canada }\end{array}$ & $21 / 9$ & $68.8 / 15$ & $\mathrm{nd} / \mathrm{nd}$ & $\mathrm{nd} / \mathrm{nd}$ & $\mathrm{nd} / \mathrm{nd}$ & 6/9/3/16 & $\mathrm{nd} / \mathrm{nd}$ & nd \\
\hline $\begin{array}{l}\text { Guan et al. [30]/ } \\
\text { 2004/China }\end{array}$ & 110/nd & nd/nd & nd/nd & $\mathrm{nd} / \mathrm{nd}$ & $8 / \mathrm{nd}$ & $\mathrm{nd} / \mathrm{nd} / \mathrm{nd} / \mathrm{nd}$ & $\mathrm{nd} / \mathrm{nd}$ & nd \\
\hline $\begin{array}{l}\text { Han et al. [31]/ } \\
\text { 2003/China }\end{array}$ & $69 / 29$ & $\mathrm{nd} / \mathrm{nd}$ & $\mathrm{nd} / \mathrm{nd}$ & $\mathrm{nd} / \mathrm{nd}$ & $\mathrm{nd} / \mathrm{nd}$ & $\mathrm{nd} / \mathrm{nd}$ & $\mathrm{nd} / \mathrm{nd}$ & nd \\
\hline $\begin{array}{l}\text { Hsiao et al. [32]/ } \\
\text { 2004/Taiwan }\end{array}$ & 346/nd & nd/nd & $\mathrm{nd} / \mathrm{nd}$ & $\mathrm{nd} / \mathrm{nd}$ & 73/nd & nd/nd/nd/nd & nd/nd & nd \\
\hline $\begin{array}{l}\text { Kumar et al. [33]/ } \\
\text { 2003/Canada }\end{array}$ & $1 / 1$ & $74 / 0$ & nd & $1 / 1$ & $1 / 1$ & nd/nd/nd/nd & $\mathrm{nd} / \mathrm{nd}$ & $\begin{array}{c}\text { cyclosporin, prednisone, insulin, } \\
\text { trimethoprim/sulfamethoxazole } \\
\text { prophylaxis }\end{array}$ \\
\hline $\begin{array}{l}\text { Lang et al. [34]/ } \\
\text { 2003/China }\end{array}$ & $3 / \mathrm{nd}$ & nd/nd & $\mathrm{nd} / \mathrm{nd}$ & $\mathrm{nd} / \mathrm{nd}$ & $\mathrm{nd} / \mathrm{nd}$ & nd/nd/nd/nd & $\mathrm{nd} / \mathrm{nd}$ & nd \\
\hline $\begin{array}{l}\text { Liu et al. [35]/ } \\
\text { 2003/China }\end{array}$ & $106 / 56$ & $36 / 10$ & $\mathrm{nd} / \mathrm{nd}$ & $\mathrm{nd} / \mathrm{nd}$ & $\mathrm{nd} / \mathrm{nd}$ & nd/nd/nd/nd & $\mathrm{nd} / \mathrm{nd}$ & steroids, antibiotics, antiviral drugs \\
\hline $\begin{array}{l}\text { Luo et al. [36]/ } \\
\text { 2003/Germany }\end{array}$ & $1 / 1$ & $54 / \mathrm{nd}$ & $1 / \mathrm{nd}$ & $1 / 1$ & $0 / 0$ & nd/nd/nd/nd & $\mathrm{nd} / \mathrm{nd}$ & ribavirin \\
\hline $\begin{array}{l}\text { Zhao et al. [37]/ } \\
\text { 2004/China }\end{array}$ & 106/nd & $\mathrm{nd} / \mathrm{nd}$ & $\mathrm{nd} / \mathrm{nd}$ & $\mathrm{nd} / \mathrm{nd}$ & $\mathrm{nd} / \mathrm{nd}$ & nd/nd/nd/nd & $\mathrm{nd} / \mathrm{nd}$ & nd \\
\hline $\begin{array}{l}\text { Yin et al. [38]/ } \\
\text { 2004/China. }\end{array}$ & 148 & $\mathrm{nd} / \mathrm{nd}$ & $\mathrm{nd} / \mathrm{nd}$ & $\mathrm{nd} / \mathrm{nd}$ & $\mathrm{nd} / \mathrm{nd}$ & $\mathrm{nd} / \mathrm{nd} / \mathrm{nd} / \mathrm{nd}$ & $\mathrm{nd} / \mathrm{nd}$ & nd \\
\hline $\begin{array}{l}\text { Yang et al. [39]/ } \\
\text { 2005/China }\end{array}$ & $168 / 72$ & $42.8 / 18.6$ & $\mathrm{nd} / \mathrm{nd}$ & $\mathrm{nd} / \mathrm{nd}$ & $\mathrm{nd} / \mathrm{nd}$ & $\mathrm{nd} / \mathrm{nd} / \mathrm{nd} / \mathrm{nd}$ & $17 / \mathrm{HBV}$ & $\begin{array}{l}\text { quinolones, macrolides, floxacin, } \\
\text { tetracycline, roxithromycin, } \\
\text { ciprofloxacin }\end{array}$ \\
\hline $\begin{array}{l}\text { Wu et al. [40]/ 2004/ } \\
\text { Taiwan }\end{array}$ & $52 / 20$ & $45 / 20$ & $\mathrm{nd} / \mathrm{nd}$ & $\mathrm{nd} / 21$ & 16/nd & $\mathrm{nd} / \mathrm{nd} / \mathrm{nd} / \mathrm{nd}$ & $8 / \mathrm{HBV}$ & nd \\
\hline $\begin{array}{l}\text { Wong et al. [41]/ } \\
\text { 2003/China }\end{array}$ & $54 / 24$ & $37.9 / 13$ & $\mathrm{nd} / \mathrm{nd}$ & $\mathrm{nd} / \mathrm{nd}$ & $\mathrm{nd} / \mathrm{nd}$ & $\mathrm{nd} / \mathrm{nd} / \mathrm{nd} / \mathrm{nd}$ & $\mathrm{nd} / \mathrm{nd}$ & $\begin{array}{c}\text { Corticosteroids and oral (or iv) ribavirin } \\
\text { cefipime, oral clarithromycin, } \\
\text { azithromycin }\end{array}$ \\
\hline
\end{tabular}


Table 1. Cont.

\begin{tabular}{|c|c|c|c|c|c|c|c|c|}
\hline $\begin{array}{l}\text { Reference/Year/ } \\
\text { Country }\end{array}$ & $\begin{array}{l}n \text { Total/ } \\
n \text { Males }\end{array}$ & $\begin{array}{c}\text { Age } \\
\text { (Mean/SD) }\end{array}$ & $\begin{array}{l}\text { Mechanical } \\
\text { Ventilation/ } \\
\text { Suppl Oxygen }\end{array}$ & $\begin{array}{l}\text { Hospitalization/ } \\
\text { ICU Stay }\end{array}$ & $\begin{array}{c}\text { Hospital } \\
\text { Mortality/ICU } \\
\text { Mortality }\end{array}$ & $\begin{array}{l}\text { Comorbidities } \\
\text { (Diabetes/ } \\
\text { Hypertension/ } \\
\text { CVD/Other) }\end{array}$ & $\begin{array}{l}\text { Preexisting } \\
\text { Liver Disease } \\
\text { (n)/Diagnosis }\end{array}$ & Intervention \\
\hline $\begin{array}{l}\text { Tong et al. [42]/ } \\
\text { 2003/China }\end{array}$ & 114/nd & nd/nd & $\mathrm{nd} / \mathrm{nd}$ & nd/nd & nd/nd & nd/nd/nd/nd & nd/nd & nd \\
\hline $\begin{array}{l}\text { Shi et al. [43]/ } \\
\text { 2005/China }\end{array}$ & $7 / 6$ & $40.43 / 13.95$ & $\mathrm{nd} / \mathrm{nd}$ & $\mathrm{nd} / \mathrm{nd}$ & $\mathrm{nd} / \mathrm{nd}$ & $\mathrm{nd} / \mathrm{nd} / \mathrm{nd} / \mathrm{nd}$ & $\mathrm{nd} / \mathrm{nd}$ & nd \\
\hline $\begin{array}{l}\text { Peiris et al. [45]/ } \\
\text { 2003/China }\end{array}$ & $50 / 22$ & $42.99 / 12.58$ & 19/nd & /nd19 & $1 / \mathrm{nd}$ & $\mathrm{nd} / \mathrm{nd} / \mathrm{nd} / \mathrm{nd}$ & $\mathrm{nd} / \mathrm{nd}$ & $\begin{array}{c}\text { Oral levofloxacin }(n=9), \\
\text { amoxicillin-clavulanate (given } \\
\text { intravenously } n=40) \text {, oseltamivir orally } \\
(n=4), \text { intravenous ceftriaxone, } \\
\text { Azithromycin, oral amantadine }(n=1), \\
\text { intravenous ribavirin, steroid }(n=49)\end{array}$ \\
\hline $\begin{array}{l}\text { Meng et al. [44]/ } \\
\text { 2003/China }\end{array}$ & $41 / 8$ & $\mathrm{nd} / \mathrm{nd}$ & $27 / 11$ & $\mathrm{nd} / \mathrm{nd}$ & $1 / \mathrm{nd}$ & $\mathrm{nd} / \mathrm{nd} / \mathrm{nd} / \mathrm{nd}$ & $\mathrm{nd} / \mathrm{nd}$ & Steroids \\
\hline \multicolumn{9}{|c|}{ MERS CoV } \\
\hline $\begin{array}{c}\text { Al Tawfiq et al. [46]/ } \\
\text { 2017/USA }\end{array}$ & $16 / n d$ & $\mathrm{nd} / \mathrm{nd}$ & $\mathrm{nd} / \mathrm{nd}$ & $15 /$ nd & nd/nd & $\mathrm{nd} / \mathrm{nd} / \mathrm{nd} / \mathrm{nd}$ & nd & nd \\
\hline $\begin{array}{l}\text { Alsaad et al. [8]/ } \\
\text { 2018/Saudi Arabia }\end{array}$ & $1 / 1$ & $33 / \mathrm{nd}$ & $1 / \mathrm{nd}$ & $1 / 1$ & $1 / 1$ & $\mathrm{nd} / \mathrm{nd} / \mathrm{nd} / 1$ & nd & $\begin{array}{l}\text { Chemotherapy, methotrexate, antibiotics } \\
\text { ifosfamide, etoposide, L-asparginase, } \\
\text { prednisolone }\end{array}$ \\
\hline $\begin{array}{l}\text { Halim et al. [47]/ } \\
\text { 2016/Egypt }\end{array}$ & $32 / 20$ & $43.99 / 13.03$ & $23 / \mathrm{nd}$ & $32 / 32$ & $14 / 14$ & $\mathrm{nd} / \mathrm{nd} / \mathrm{nd} / 31$ & nd & nd \\
\hline $\begin{array}{l}\text { Ling et al. [48]/ } \\
\text { 2015/China }\end{array}$ & $1 / \mathrm{nd}$ & 43/nd & $1 / \mathrm{nd}$ & $1 / \mathrm{nd}$ & $\mathrm{nd} / \mathrm{nd}$ & $\mathrm{nd} / \mathrm{nd} / \mathrm{nd} / \mathrm{nd}$ & nd & Ribavirin, ceftriaxone, meropenem \\
\hline $\begin{array}{l}\text { Kapoor et al. [49]/ } \\
\text { 2014/USA }\end{array}$ & $1 / 1$ & $65 / \mathrm{nd}$ & $0 /$ nd & $1 / 0$ & $0 / 0$ & $\mathrm{nd} / 1 / 1 / 1$ & nd & $\begin{array}{c}\text { vancomycin, piperacillin/, ceftriaxone } \\
\text { tazobactam, levofloxacin, linezolid, } \\
\text { furosemide }\end{array}$ \\
\hline $\begin{array}{l}\text { Yousefi et al. [50]/ } \\
\text { 2017/Iran }\end{array}$ & $5 / 1$ & $49.6 / 10.52$ & $\mathrm{nd} / \mathrm{nd}$ & $4 / \mathrm{nd}$ & $3 / 3$ & $\mathrm{nd} / 1 / \mathrm{nd} / 1$ & nd & $\begin{array}{l}\text { PT1: azithromycin, ceftriaxone, } \\
\text { meropenem, vancomycin, oseltamivir; } \\
\text { PT2: levofloxacin, ceftriaxone, } \\
\text { azithromycin, oseltamivir; PT3: no } \\
\text { drugs, P4: no data (pt. died in ICU), P5: } \\
\text { meropenem and vancomycin, } \\
\text { oseltamivir }\end{array}$ \\
\hline $\begin{array}{l}\text { Sherbini et al. [51]/ } \\
\text { 2017/Saudi Arabia }\end{array}$ & $29 / 20$ & $45.49 / 12.22$ & 9/nd & $\mathrm{nd} / \mathrm{nd}$ & 10/nd & $9 / \mathrm{nd} / \mathrm{nd} / 8$ & nd & $\begin{array}{c}\text { Meropenem }(n=20), \text { linezolid }(n=17), \\
\text { levofloxacin }(n=15), \text { piperacillin } \\
(n=15) \text {, ribavirin }(n=10) \text {, azithromycin } \\
(n=19) \text {, interferon }(n=19) \text {, steroids } \\
(n=29)\end{array}$ \\
\hline
\end{tabular}


Table 1. Cont.

\begin{tabular}{|c|c|c|c|c|c|c|c|c|}
\hline $\begin{array}{l}\text { Reference/Year/ } \\
\text { Country }\end{array}$ & $\begin{array}{l}n \text { Total/ } \\
n \text { Males }\end{array}$ & $\begin{array}{c}\text { Age } \\
\text { (Mean/SD) }\end{array}$ & $\begin{array}{l}\text { Mechanical } \\
\text { Ventilation/ } \\
\text { Suppl Oxygen }\end{array}$ & $\begin{array}{l}\text { Hospitalization/ } \\
\text { ICU Stay }\end{array}$ & $\begin{array}{l}\text { Hospital } \\
\text { Mortality/ICU } \\
\text { Mortality }\end{array}$ & $\begin{array}{l}\text { Comorbidities } \\
\text { (Diabetes/ } \\
\text { Hypertension/ } \\
\text { CVD/Other) }\end{array}$ & $\begin{array}{l}\text { Preexisting } \\
\text { Liver Disease } \\
\text { (n)/Diagnosis }\end{array}$ & Intervention \\
\hline $\begin{array}{l}\text { Saad et al. [52]/ } \\
\text { 2014/Saudi Arabia }\end{array}$ & $70 / 46$ & $61 * /(1-90) Z$ & 49/nd & $\mathrm{nd} / 49$ & $42 / \mathrm{nd}$ & nd/nd/nd/nd & nd & nd \\
\hline $\begin{array}{c}\mathrm{Ng} \text { et al. }[53] / \\
\text { 2014/United Arab } \\
\text { Emirates }\end{array}$ & $1 / 1$ & 45 & $1 / \mathrm{nd}$ & $\mathrm{nd} / \mathrm{nd}$ & $\mathrm{nd} / \mathrm{nd}$ & nd/nd/nd/nd & nd & $\begin{array}{c}\text { Prednisolone, paracetamol, levofloxacin, } \\
\text { oseltamivir, ceftriaxone, azithromycin, } \\
\text { hydrocortisone intravenously }\end{array}$ \\
\hline
\end{tabular}

\# $\min$ and max * median; $\mathrm{Z}$ interquartile range (IQR); nd, no data; HBV, hepatitis B virus; SD, standard deviation; ICU, intensive care unit; CVD, cardiovascular disease; SARS CoV-1,

severe acute respiratory syndrome coronavirus 1; SARC-CoV-2, severe acute respiratory syndrome coronavirus 2; MERS-CoV, Middle East respiratory syndrome coronavirus.

Table 2. Liver-related outcomes in presented studies.

\begin{tabular}{|c|c|c|c|c|c|c|c|c|c|c|}
\hline Reference & $n$ Total & $\begin{array}{l}\text { AST (Mean) } \\
\text { ALT (Mean) } \\
\text { LDH (Mean) }\end{array}$ & $\begin{array}{l}\text { Abnormal AST (n) } \\
\text { Abnormal ALT (n) } \\
\text { Abnormal LDH (n) }\end{array}$ & $\begin{array}{c}\text { Bilirubin (Mean) } \\
\text { ALP (Mean) } \\
\text { Creatinine } \\
\text { (Mean) }\end{array}$ & $\begin{array}{c}\text { Abnormal } \\
\text { Bilirubin }(n) \\
\text { Abnormal ALP }(n) \\
\text { Abnormal } \\
\text { Creatinine }(n)\end{array}$ & $\begin{array}{l}\text { Total } \\
\text { Protein } \\
\text { (Mean) } \\
\text { Albumin } \\
\text { (Mean) }\end{array}$ & $\begin{array}{c}\text { Abnormal } \\
\text { Tot. Protein } \\
(n) \\
\text { Abnormal } \\
\text { Albumin }(n)\end{array}$ & $\begin{array}{l}\text { Prothrombin } \\
\text { Time } \\
\text { (Mean) } \\
\text { INR (Mean) }\end{array}$ & $\begin{array}{c}\text { Abnormal } \\
\text { Prothrombin } \\
\text { Time }(n) \\
\text { Abnormal } \\
\text { INR }(n)\end{array}$ & $\begin{array}{l}\text { Abnormal } \\
\text { Liver } \\
\text { Function } \\
(n / n \text { Total) }\end{array}$ \\
\hline \multicolumn{11}{|c|}{ SARS CoV-2 } \\
\hline $\begin{array}{l}\text { Chen et al. [16]/ } \\
\text { 2020/China }\end{array}$ & 99 & nd & $\begin{array}{l}\text { AST: } 35 \\
\text { ALT: } 28 \\
\text { LDH: } 75\end{array}$ & nd & $\begin{array}{l}\text { BIL: } 18 \\
\text { CR: } 24\end{array}$ & nd & nd & nd & nd & nd \\
\hline $\begin{array}{c}\text { Chuan et al. [17]/ } \\
\text { 2020/China }\end{array}$ & 32 & $\begin{array}{l}\text { AST (U/L): } 24.75 \\
\text { ALT (U/L): } 26.98\end{array}$ & nd & BIL (mmol/L): 16.4 & nd & $\begin{array}{c}\operatorname{ALB}(\mathrm{g} / \mathrm{L}): \\
39\end{array}$ & nd & nd & nd & nd \\
\hline [2]/ 2020/China & 1099 & nd & $\begin{array}{l}\text { AST: } 168 \\
\text { ALT: } 158 \\
\text { LDH: } 277\end{array}$ & nd & $\begin{array}{l}\text { BIL: } 76 \\
\text { CR: } 12\end{array}$ & nd & nd & nd & nd & nd \\
\hline $\begin{array}{l}\text { Wang et al. [12]/ } \\
\text { 2020/China }\end{array}$ & 138 & $\begin{array}{l}\text { AST (U/L): } 31^{*} \\
\text { ALT (U/L): } 24^{*} \\
\text { LDH (U/L): } 261 *\end{array}$ & nd & $\begin{array}{l}\text { BIL }(\mathrm{mmol} / \mathrm{L}): 9.8^{*} \\
\text { CR }(\mu \mathrm{mol} / \mathrm{L}): 72^{*}\end{array}$ & nd & nd & nd & PT (s): $13 *$ & nd & nd \\
\hline $\begin{array}{c}\text { Zhou et al. [18]/ } \\
\text { 2020/China }\end{array}$ & 191 & $\begin{array}{l}\text { ALT (U/L): } 30^{*} \\
\text { LDH (U/L): } 300^{*}\end{array}$ & $\begin{array}{l}\text { ALT: } 59 \\
\text { LDH: } 123\end{array}$ & nd & CR: 8 & $\begin{array}{c}\text { ALB }(\mathrm{g} / \mathrm{L}): \\
32.3^{*}\end{array}$ & nd & PT (s): $11.6^{*}$ & PT: 182 & nd \\
\hline $\begin{array}{l}\text { Zhang et al. [19]/ } \\
\text { 2020/China }\end{array}$ & 56 & nd & nd & nd & ALP: 1 & nd & nd & nd & nd & $16 / 56$ \\
\hline
\end{tabular}


Table 2. Cont

\begin{tabular}{|c|c|c|c|c|c|c|c|c|c|c|}
\hline Reference & $n$ Total & $\begin{array}{l}\text { AST (Mean) } \\
\text { ALT (Mean) } \\
\text { LDH (Mean) }\end{array}$ & $\begin{array}{c}\text { Abnormal AST }(n) \\
\text { Abnormal ALT }(n) \\
\text { Abnormal LDH }(n)\end{array}$ & $\begin{array}{l}\text { Bilirubin (Mean) } \\
\text { ALP (Mean) } \\
\text { Creatinine } \\
\text { (Mean) }\end{array}$ & $\begin{array}{c}\text { Abnormal } \\
\text { Bilirubin }(n) \\
\text { Abnormal ALP }(n) \\
\text { Abnormal } \\
\text { Creatinine }(n)\end{array}$ & $\begin{array}{l}\text { Total } \\
\text { Protein } \\
\text { (Mean) } \\
\text { Albumin } \\
\text { (Mean) }\end{array}$ & $\begin{array}{c}\text { Abnormal } \\
\text { Tot. Protein } \\
(n) \\
\text { Abnormal } \\
\text { Albumin }(n)\end{array}$ & $\begin{array}{l}\text { Prothrombin } \\
\text { Time } \\
\text { (Mean) } \\
\text { INR (Mean) }\end{array}$ & $\begin{array}{c}\text { Abnormal } \\
\text { Prothrombin } \\
\text { Time }(n) \\
\text { Abnormal } \\
\text { INR }(n)\end{array}$ & $\begin{array}{c}\text { Abnorma } \\
\text { Liver } \\
\text { Function } \\
(n / n \text { Total })\end{array}$ \\
\hline $\begin{array}{l}\text { Yang et al. [20]/ } \\
\text { 2020/China }\end{array}$ & 52 & nd & nd & $\begin{array}{c}\text { BIL }(\mu \mathrm{mol} / \mathrm{L}): \\
17.04 \\
\text { CR }(\mu \mathrm{mol} / \mathrm{L}): 79\end{array}$ & nd & nd & nd & PT (s): 12.3 & nd & nd \\
\hline $\begin{array}{l}\text { Xu et al. [21]/ } \\
\text { 2020/China }\end{array}$ & 62 & $\begin{array}{c}\text { AST(U/L): } 26^{*} \\
\text { ALT (U/L):22* } \\
\text { LDH (U/L): } 205^{*}\end{array}$ & $\begin{array}{l}\text { AST: } 10 \\
\text { LDH: } 17\end{array}$ & $\mathrm{CR}(\mu \mathrm{mol} / \mathrm{L}): 72$ & CR: 3 & nd & nd & nd & nd & nd \\
\hline $\begin{array}{l}\text { Wu et al. [22]/ } \\
\text { 2020/China }\end{array}$ & 80 & $\begin{array}{l}\text { AST (U/L): } 30^{*} \\
\text { ALT (U/L): } 24^{*} \\
\text { LDH (U/L): } 226^{*}\end{array}$ & $\begin{array}{l}\text { AST: } 3 \\
\text { ALT: } 3 \\
\text { LDH: } 17\end{array}$ & $\begin{array}{c}\mathrm{BIL}(\mu \mathrm{mol} / \mathrm{L}): 6.6 \\
\mathrm{CR}(\mu \mathrm{mol} / \mathrm{L}): 78\end{array}$ & $\begin{array}{l}\text { BIL: } 1 \\
\text { CR: } 2\end{array}$ & $\begin{array}{l}\operatorname{ALB}(g / L): \\
38.3^{*}\end{array}$ & ALB: 2 & PT (s): 10.8 & nd & nd \\
\hline $\begin{array}{l}\text { Shi et al. [23]/ } \\
\text { 2020/China }\end{array}$ & 81 & $\begin{array}{l}\text { AST (U/L): } 40.8 \\
\text { ALT (U/L): } 46.2\end{array}$ & AST: 43 & $\begin{array}{l}\text { BIL }(\mu \mathrm{mol} / \mathrm{L}): 11.9 \\
\text { CR }(\mu \mathrm{mol} / \mathrm{L}): 75.4\end{array}$ & nd & $\begin{array}{l}\text { ALB }(\mathrm{g} / \mathrm{L}): \\
32.9\end{array}$ & nd & PT (s): 10.7 & nd & nd \\
\hline $\begin{array}{l}\text { Jin et al. [4]/ } \\
\text { 2020/China }\end{array}$ & 651 & $\begin{array}{c}\text { AST (U/L): } 29.35 \\
\text { (g)/24.2 (ng)* } \\
\text { ALT (U/L): } \\
\text { 25(g)/21.5 (ng) } \\
\text { LDH (U/L): } \\
\text { 229(g)/210 (ng) }\end{array}$ & nd & $\begin{array}{c}\mathrm{BIL}(\mu \mathrm{mol} / \mathrm{L}): 10 \\
(\mathrm{~g}) / 9.6(\mathrm{ng}) \\
\mathrm{CR}(\mu \mathrm{mol} / \mathrm{L}): 66.0 \\
(\mathrm{~g}) / 66.0(\mathrm{ng})\end{array}$ & nd & $\begin{array}{l}\text { ALB (g/L): } \\
40.13(\mathrm{~g}) / 41.5 \\
\text { (ng) }\end{array}$ & nd & nd & $\begin{array}{l}\text { INR: } 1.03 \\
\text { (g)/1.02 (ng) }\end{array}$ & nd \\
\hline \multicolumn{11}{|c|}{ SARS CoV-1 } \\
\hline $\begin{array}{l}\text { Chan et al. [24]/ } \\
\text { 2004/China }\end{array}$ & 118 & ALT (U/L): 25.5 & ALT: 25 & $\mathrm{CR}(\mu \mathrm{mol} / \mathrm{L}): 85$ & nd & nd & nd & PT (s): $11.2 *$ & nd & nd \\
\hline $\begin{array}{c}\text { Chan et al. [25]/ } \\
\text { 2005/China }\end{array}$ & 294 & nd & ALT: 52 & nd & ALP: 40 & nd & nd & nd & nd & nd \\
\hline $\begin{array}{l}\text { Chau et al. [13]/ } \\
\text { 2004/China }\end{array}$ & 3 & ALT (U/L): 165X & nd & $\operatorname{BIL}(\mu \mathrm{mol} / \mathrm{L}): 7.3 \mathrm{X}$ & nd & $\begin{array}{l}\operatorname{ALB}(\mathrm{g} / \mathrm{L}): \\
32 \mathrm{Y}\end{array}$ & nd & nd & nd & nd \\
\hline $\begin{array}{l}\text { Chen et al. [26]/ } \\
\text { 2003/China }\end{array}$ & 7 & nd & nd & nd & nd & nd & nd & nd & nd & nd \\
\hline $\begin{array}{l}\text { Cui et al. [27]/ } \\
\text { 2004/China }\end{array}$ & 182 & nd & $\begin{array}{l}\text { ALT: } 89 \\
\text { AST: } 89 \\
\text { LDH: } 76\end{array}$ & nd & nd & nd & nd & nd & nd & nd \\
\hline $\begin{array}{l}\text { Ding et al. [28]/ } \\
\text { 2003/China }\end{array}$ & 3 & nd & nd & nd & nd & nd & nd & nd & nd & nd \\
\hline $\begin{array}{l}\text { Farcas et al. [29]/ } \\
\text { 2005/Canada }\end{array}$ & 21 & nd & nd & nd & nd & nd & nd & nd & nd & nd \\
\hline
\end{tabular}


Table 2. Cont.

\begin{tabular}{|c|c|c|c|c|c|c|c|c|c|c|}
\hline Reference & $n$ Total & $\begin{array}{l}\text { AST (Mean) } \\
\text { ALT (Mean) } \\
\text { LDH (Mean) }\end{array}$ & $\begin{array}{l}\text { Abnormal AST (n) } \\
\text { Abnormal ALT }(n) \\
\text { Abnormal LDH }(n)\end{array}$ & $\begin{array}{l}\text { Bilirubin (Mean) } \\
\text { ALP (Mean) } \\
\text { Creatinine } \\
\text { (Mean) }\end{array}$ & $\begin{array}{c}\text { Abnormal } \\
\text { Bilirubin }(n) \\
\text { Abnormal ALP }(n) \\
\text { Abnormal } \\
\text { Creatinine }(n)\end{array}$ & $\begin{array}{l}\text { Total } \\
\text { Protein } \\
\text { (Mean) } \\
\text { Albumin } \\
\text { (Mean) }\end{array}$ & $\begin{array}{c}\text { Abnormal } \\
\text { Tot. Protein } \\
(n) \\
\text { Abnormal } \\
\text { Albumin }(n)\end{array}$ & $\begin{array}{l}\text { Prothrombin } \\
\text { Time } \\
\text { (Mean) } \\
\text { INR (Mean) }\end{array}$ & $\begin{array}{c}\text { Abnormal } \\
\text { Prothrombin } \\
\text { Time }(n) \\
\text { Abnormal } \\
\text { INR }(n)\end{array}$ & $\begin{array}{c}\text { Abnorma } \\
\text { Liver } \\
\text { Function } \\
(n / n \text { Total })\end{array}$ \\
\hline $\begin{array}{l}\text { Guan et al. [30]/ } \\
\text { 2004/China }\end{array}$ & 110 & $\begin{array}{c}\text { ALT (U/L): } \\
\text { 91.61X } \\
\text { AST (U/L): } \\
78.68 X \\
\text { LDH (U/L): } \\
429.69 X\end{array}$ & nd & $\begin{array}{l}\mathrm{BIL}(\mu \mathrm{mol} / \mathrm{L}): \\
11.67 \mathrm{X}\end{array}$ & ALP: 0 & $\begin{array}{l}\mathrm{ALB}(\mathrm{g} / \mathrm{L}): \\
34.4\end{array}$ & nd & nd & nd & nd \\
\hline $\begin{array}{l}\text { Han et al. [31]/ } \\
\text { 2003/China }\end{array}$ & 69 & nd & nd & nd & nd & nd & nd & nd & nd & $37 /$ nd \\
\hline $\begin{array}{l}\text { Hsiao et al. [32]/ } \\
\text { 2004/Taiwan }\end{array}$ & 346 & nd & nd & nd & nd & nd & nd & nd & nd & nd \\
\hline $\begin{array}{l}\text { Kumar et al. [33]/ } \\
\text { 2003/Canada }\end{array}$ & 1 & AST (U/L):51 & ALT: 1 & nd & BIL: 1 & nd & nd & nd & nd & nd \\
\hline $\begin{array}{l}\text { Lang et al. [34]/ } \\
\text { 2003/China }\end{array}$ & 3 & nd & nd & nd & nd & nd & nd & nd & nd & nd \\
\hline $\begin{array}{l}\text { Liu et al. [35]/ } \\
\text { 2003/China }\end{array}$ & 106 & nd & ALT: 8 & nd & nd & nd & nd & nd & nd & nd \\
\hline $\begin{array}{l}\text { Luo et al. [36]/ } \\
\text { 2003/Germany }\end{array}$ & 1 & $\begin{array}{c}\text { ALT (U/L): } \\
425.5 \mathrm{X} \\
\text { AST (U/L): } 319 \mathrm{X}\end{array}$ & LDH: 0 & nd & CR: 0 & nd & nd & nd & nd & nd \\
\hline $\begin{array}{c}\text { Zhao et al. [37]/ } \\
\text { 2004/China }\end{array}$ & 106 & nd & $\begin{array}{l}\text { ALT: } 106 \\
\text { AST: } 68\end{array}$ & nd & nd & nd & $\begin{array}{c}\text { TP: } 0 \\
\text { ALB: } 122\end{array}$ & nd & nd & nd \\
\hline $\begin{array}{l}\text { Yin et al. [38]/ } \\
\text { 2004/China }\end{array}$ & 148 & nd & nd & nd & nd & nd & nd & nd & nd & 148 \\
\hline $\begin{array}{l}\text { Yang et al. [39]/ } \\
\text { 2005/China }\end{array}$ & 168 & $\begin{array}{c}\text { ALT (U/L): } \\
111.32 X \\
\text { AST (U/L): } \\
48.95 X\end{array}$ & ALT:118 & $\begin{array}{c}\text { BIL: }(\mu \mathrm{mol} / \mathrm{L}): \\
10.41 \mathrm{X}\end{array}$ & nd & $\begin{array}{l}\mathrm{ALB}(\mathrm{mg} / \mathrm{L}): \\
34.26 \mathrm{Y}\end{array}$ & nd & nd & nd & nd \\
\hline $\begin{array}{l}\text { Wu et al. [40]/ } \\
\text { 2004/ Taiwan }\end{array}$ & 52 & $\begin{array}{c}\text { ALT (U/L): } \\
\text { 86.19X } \\
\text { AST (U/L): } 69.05 \\
X \\
\end{array}$ & $\begin{array}{l}\text { ALT: } 28 \\
\text { AST: } 28\end{array}$ & nd & nd & nd & nd & nd & nd & nd \\
\hline $\begin{array}{l}\text { Wong et al. [41]/ } \\
\text { 2003/China }\end{array}$ & 54 & $\begin{array}{l}\text { ALT (U/L): } 95.7 \mathrm{X} \\
\text { AST }(\mathrm{U} / \mathrm{L}): 62.8 \mathrm{X}\end{array}$ & ALT:41X & $\begin{array}{c}\text { BIL: }(\mu \mathrm{mol} / \mathrm{L}): \\
11.1 \mathrm{X} \\
\text { ALP (U/L): } 72.6 \mathrm{X}\end{array}$ & nd & $\begin{array}{l}\operatorname{ALB}(\mathrm{g} / \mathrm{L}): \\
33.2 \mathrm{Y}\end{array}$ & nd & nd & 61 & nd \\
\hline $\begin{array}{l}\text { Tong et al. [42]/ } \\
\text { 2003/China }\end{array}$ & 114 & nd & nd & nd & nd & nd & nd & nd & nd & $84 /$ nd \\
\hline
\end{tabular}


Table 2. Cont

\begin{tabular}{|c|c|c|c|c|c|c|c|c|c|c|}
\hline Reference & $n$ Total & $\begin{array}{l}\text { AST (Mean) } \\
\text { ALT (Mean) } \\
\text { LDH (Mean) }\end{array}$ & $\begin{array}{c}\text { Abnormal AST }(n) \\
\text { Abnormal ALT }(n) \\
\text { Abnormal LDH }(n)\end{array}$ & $\begin{array}{l}\text { Bilirubin (Mean) } \\
\text { ALP (Mean) } \\
\text { Creatinine } \\
\text { (Mean) }\end{array}$ & $\begin{array}{c}\text { Abnormal } \\
\text { Bilirubin }(n) \\
\text { Abnormal ALP }(n) \\
\text { Abnormal } \\
\text { Creatinine }(n)\end{array}$ & $\begin{array}{l}\text { Total } \\
\text { Protein } \\
\text { (Mean) } \\
\text { Albumin } \\
\text { (Mean) }\end{array}$ & $\begin{array}{c}\text { Abnormal } \\
\text { Tot. Protein } \\
(n) \\
\text { Abnormal } \\
\text { Albumin }(n)\end{array}$ & $\begin{array}{l}\text { Prothrombin } \\
\text { Time } \\
\text { (Mean) } \\
\text { INR (Mean) }\end{array}$ & $\begin{array}{c}\text { Abnormal } \\
\text { Prothrombin } \\
\text { Time }(n) \\
\text { Abnormal } \\
\text { INR }(n)\end{array}$ & $\begin{array}{c}\text { Abnorma } \\
\text { Liver } \\
\text { Function } \\
(n / n \text { Total })\end{array}$ \\
\hline $\begin{array}{l}\text { Shi et al. [43]/ } \\
\text { 2005/China }\end{array}$ & 7 & nd & AST: 4 & nd & nd & nd & nd & nd & nd & nd \\
\hline $\begin{array}{l}\text { Peiris et al. [45]/ } \\
\text { 2003/China }\end{array}$ & 50 & $\operatorname{ALT}(\mathrm{U} / \mathrm{L}): 63 *$ & ALT: 17 & nd & nd & $\begin{array}{c}\text { ALB }(\mathrm{g} / \mathrm{L}): \\
37\end{array}$ & ALB: 34 & nd & nd & $17 /$ nd \\
\hline $\begin{array}{l}\text { Meng et al. [44]/ } \\
\text { 2003/China }\end{array}$ & 41 & nd & nd & nd & nd & nd & nd & nd & nd & $27 / \mathrm{nd}$ \\
\hline \multicolumn{11}{|c|}{ MERS CoV } \\
\hline $\begin{array}{c}\text { Al } \\
\text { Tawfiq et al. [46]/ } \\
\text { 2017/USA }\end{array}$ & 16 & $\begin{array}{c}\text { AST (U/L): } 661 \mathrm{X} \\
\text { ALT (U/L): } 476 \mathrm{X} \\
\text { LDH (U/L): } \\
1825.8 \mathrm{X}\end{array}$ & nd & $\begin{array}{l}\text { BIL }(\mu \mathrm{mol} / \mathrm{L}): 21 X \\
\text { ALP (U/L): } 257.3 X \\
\text { CR (mg/dL): } 3.8 X\end{array}$ & nd & nd & nd & nd & nd & nd \\
\hline $\begin{array}{l}\text { Alsaad et al. [8]/ } \\
\text { 2018/Saudi Arabia }\end{array}$ & 1 & nd & nd & nd & nd & nd & nd & nd & nd & $0 / 1$ \\
\hline $\begin{array}{c}\text { Halim et al. [47]/ } \\
\text { 2016/Egypt }\end{array}$ & 32 & nd & nd & nd & nd & nd & nd & nd & nd & nd \\
\hline $\begin{array}{l}\text { Ling et al. [48]/ } \\
\text { 2015/China }\end{array}$ & 1 & nd & nd & nd & nd & nd & nd & nd & nd & nd \\
\hline $\begin{array}{c}\text { Kapoor et al. [49]/ } \\
\text { 2014/USA }\end{array}$ & 1 & $\begin{array}{l}\text { AST (U/L): } 95 \\
\text { ALT (U/L): } 80\end{array}$ & nd & $\begin{array}{c}\text { BIL (mg/dL): } 1 \\
\text { ALP (U/L) } 270 \\
\text { CR (mg/dL): } 0.75\end{array}$ & nd & nd & nd & nd & nd & nd \\
\hline $\begin{array}{c}\text { Yousefi et al. [50]/ } \\
\text { 2017/Iran }\end{array}$ & 5 & $\begin{array}{c}\text { AST (U/L): } 60.7 \\
\text { ALT (U/L): } 35.75\end{array}$ & $\begin{array}{l}\text { AST: } 3 \\
\text { ALT: } 2\end{array}$ & $\mathrm{CR}(\mathrm{mg} / \mathrm{dL}): 0.77$ & nd & nd & nd & $\begin{array}{l}\text { PT: } 13.65 \\
\text { INR: } 1.1\end{array}$ & $\begin{array}{l}\text { PT: } 2 \\
\text { INR: } 1\end{array}$ & nd \\
\hline $\begin{array}{l}\text { Sherbini et al. [51]/ } \\
\text { 2017/Saudi Arabia }\end{array}$ & 29 & $\begin{array}{l}\text { AST (U/L): } 86.3 \\
\text { ALT (U/L): } 98.4\end{array}$ & nd & $\begin{array}{c}\text { BIL }(\mu \mathrm{mol} / \mathrm{L}): \\
16.64 \\
\text { CR }(\mu \mathrm{mol} / \mathrm{L}): 225\end{array}$ & nd & nd & nd & nd & nd & nd \\
\hline $\begin{array}{l}\text { Saad et al. [52]/ } \\
\text { 2014/Saudi Arabia }\end{array}$ & 70 & $\begin{array}{c}\text { AST (U/L): } 112 * \\
\text { XALT (U/L): } \\
54{ }^{*} \mathrm{X}\end{array}$ & nd & $\begin{array}{c}\text { BIL }(\mu \mathrm{mol} / \mathrm{L}): 17^{*} \\
\text { XALP }(\mathrm{U} / \mathrm{L}): 145^{*} \\
\text { XCR }(\mu \mathrm{mol} / \mathrm{L}): \\
251.5^{*} \mathrm{X}\end{array}$ & nd & $\begin{array}{c}\text { ALB } \\
(\mathrm{mg} / \mathrm{dL}): \\
21 * \mathrm{Y}\end{array}$ & nd & nd & nd & $22 / 70$ \\
\hline $\begin{array}{c}\text { Ng et al. [53]/ } \\
\text { 2014/United Arab } \\
\text { Emirates }\end{array}$ & 1 & $\begin{array}{l}\text { AST (U/L): } 51 \\
\text { ALT (U/L): } 28\end{array}$ & nd & $\mathrm{CR}(\mathrm{mg} / \mathrm{dL}): 0.9$ & nd & nd & nd & $\begin{array}{l}\text { PT: } 12 \\
\text { INR: } 1.1\end{array}$ & nd & nd \\
\hline
\end{tabular}

* median; $X$, highest measurement during the study; $\mathrm{Y}$, lowest measurement during the study; nd, not determined; AST, aspartate aminotransferase; ALT, alanine aminotrasfesrase; LDH, lactate dehydrogenase; ALP, alkaline phosphatase; INR, international normalized ratio; BIL, bilirubin; ALB, albumin; CR, creatinine; PT, prothrombin time. 
Table 3. Histopathological findings within the livers of patients infected with coronaviruses.

\begin{tabular}{|c|c|c|c|}
\hline Reference & $\begin{array}{l}\text { Post-Mortem } \\
\text { Study }(\mathrm{Y} / \mathrm{N})\end{array}$ & $\begin{array}{l}\text { Type of } \\
\text { Coronavirus }\end{array}$ & Histopathology Cases (in Words) \\
\hline [24] & $\mathrm{N}$ & SARS-CoV-1 & - no acute changes, no necrosis \\
\hline [13] & $\mathrm{N}$ & SARS-CoV-1 & $\begin{array}{l}\text { - mild lobular activities with occasional acidophilic } \\
\text { bodies and prominent Kupffer cell } \\
\text { - smildly inflamed portal tracts with lymphocytic } \\
\text { infiltration }\end{array}$ \\
\hline [26] & $\mathrm{Y}$ & SARS-CoV-1 & $\begin{array}{l}\text { - massive necrosis ( } 1 \text { case }) \\
\text { - nodular cirrhosis ( } 1 \text { case) }\end{array}$ \\
\hline [28] & $\mathrm{Y}$ & SARS-CoV-1 & $\begin{array}{l}\text { - dissociation of hepatocyte cords, together with fatty } \\
\text { degeneration and focal necrosis ( } 1 \text { case) } \\
\text { - massive central necrosis of hepatocytes ( } 2 \text { cases) } \\
\text { - the vascular walls with edema and infiltration of } \\
\text { monocytes and lymphocytes }\end{array}$ \\
\hline [29] & $\mathrm{Y}$ & SARS-CoV-1 & $\begin{array}{l}\text { - minor inflammatory changes observed in the liver on } \\
\text { microscopic examination }\end{array}$ \\
\hline [30] & $\mathrm{N}$ & SARS-CoV-1 & $\begin{array}{l}\text { - non-specific inflammation in the liver in biopsy } \\
\text { - non-specific hepatitis in postmortem biopsy }\end{array}$ \\
\hline [32] & $\mathrm{N}$ & SARS-CoV-1 & $\begin{array}{l}\text { - no specific pathological change in the gastrointestinal } \\
\text { tract }\end{array}$ \\
\hline [34] & $\mathrm{Y}$ & SARS-CoV-1 & $\begin{array}{l}\text { - hydropic degeneration } \\
\text { - fatty degeneration } \\
\text { - interstitial cell proliferation }\end{array}$ \\
\hline [39] & $\mathrm{N}$ & SARS-CoV-1 & $\begin{array}{l}\text { - hydropic degeneration } \\
\text { - } \text { steatosis } \\
\text { - focal necrosis }(n=4)\end{array}$ \\
\hline [43] & Y & SARS-CoV-1 & $\begin{array}{l}\text { - } \text { mild fatty-acid degeneration } \\
\text { - } \text { mild congestion } \\
\text { - central lobular necrosis }\end{array}$ \\
\hline [8] & $\mathrm{Y}$ & MERS-CoV & $\begin{array}{l}\text { - mild chronic lymphocytic portal inflammation } \\
\text { - reactive parenchyma with mild cellular hydropic } \\
\text { degeneration } \\
\text { - rare multinucleated hepatocytes and mild disarray of } \\
\text { the hepatic plates } \\
\text { - mild sinusoidal lymphocytosis and small } \\
\text { necroinflammatory foci in the hepatic lobules } \\
\text { - congestion, hemorrhage and focal perivenular loss of } \\
\text { hepatocytes } \\
\text { - macrovesicular perivenular steatotic change, } \\
\text { sinusoidal congestion, hemorrhage and focal perivenular } \\
\text { loss of hepatocytes }\end{array}$ \\
\hline [53] & Y & MERS-CoV & $\begin{array}{l}\text { - } \text { moderate steatosis } \\
\text { - } \text { scattered calcifications } \\
\text { inflammation }\end{array}$ \\
\hline
\end{tabular}

SARS-CoV-1, severe acute respiratory syndrome coronavirus 1; MERS-CoV, Middle East respiratory syndrome coronavirus; $\mathrm{N}$, no; $\mathrm{Y}$, yes. 


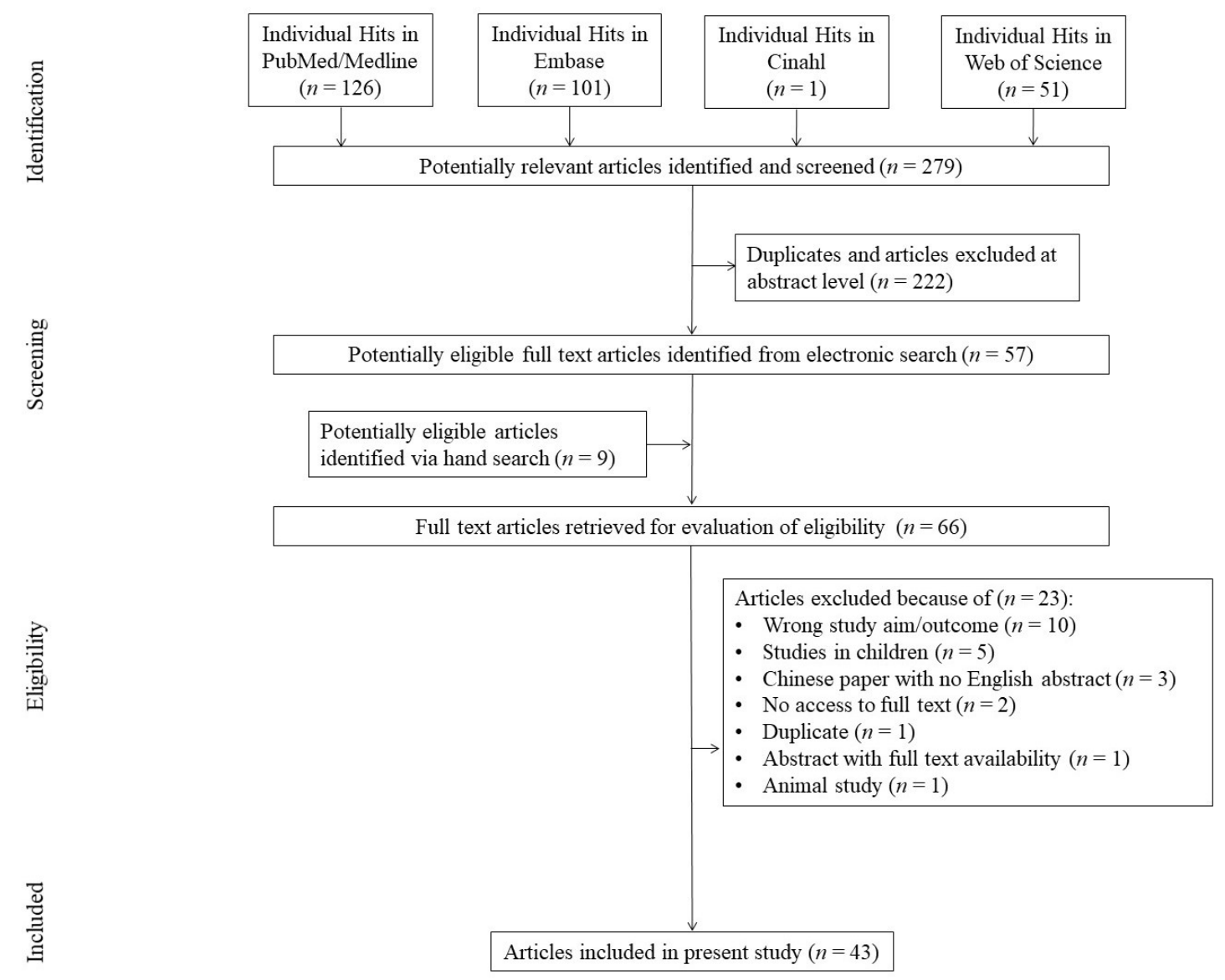

Figure 1. Study and sample characteristics.

\section{Discussion}

A number of studies have documented SARS infection associated with liver injury as manifested by mild and moderate elevation of ALT and/or AST at the onset of the disease $[11,19,54]$. Angiotensin-converting enzyme 2 (ACE2) has been established as a membrane receptor for SARS-CoV cell entry [55]. ACE2 was shown to be abundantly expressed in several cell types, including not only lung type II alveolar cells (AT2) but also monocytes and vascular endothelial cells [56]. Recently, Meng-Yuan et al. compared ACE2 expression levels across 31 human tissues using the datasets from the Genotype-Tissue Expression (GTEx) project and the Cancer Genome Atlas (TCGA) program. The researchers were able to report highest ACE2 expression in the small intestine, testis, kidneys, heart, thyroid and adipose tissue and medium expression in the lungs, colon, liver, bladder and adrenal gland [57]. The study by Paizis et al. [58] using immunohistochemistry confirmed ACE2 expression in hepatocytes of healthy human liver which increased in chronic liver diseases and during hypoxia. Therefore, gastrointestinal manifestations of the disease are likely a result of alterations of the gut-liver and gut-pulmonary axes. As documented by Spadoni et al. [59] the gut vascular barrier (GVB) is vulnerable to noxious infectious agents with the end result of systemic dissemination of pathogens. In patients with coeliac disease (CD) with elevated serum transaminases GVB alterations may be responsible for liver damage [59]. Although gut barrier disruption has not been reported to be a pre requisite for nonalcoholic steatohepatitis (NASH) development and belongs to the 'multiple hit' pathogenesis of disease progression [60], more recent observations in preclinical models have shown that disruption of epithelial and vascular barriers in the intestine were early events reported in NASH [61]. Gut-liver axis deterioration has also been described in patients with chronic liver diseases who develop sepsis and multiorgan failure $[62,63]$. Zhou et al. in their recent retrospective cohort study of 191 patients reported that more than half of hospitalized patients developed sepsis as a common 
complication of SARS-CoV-2 infection [18]. However, data on the pathogenesis of sepsis in COVID-19 patients and the potential role of gut-liver axis and hepatic dysfunction remain unknown.

\subsection{COVID-19 Patients and Liver Injury}

In our current study we evaluated the data on laboratory and liver pathology parameters of 4591 persons, infected with SARS CoV-2 $(n=2541)$, followed by SARS CoV-1 $(n=1894)$ and MERS $(n=156)$. All the studies analyzed were retrospective and/or case reports. The mean/median age of all study patients ranged between 33 and 45.21 years. Main comorbidities reported in 14 studies included hypertension (HTN) in 306, diabetes mellitus (DM) in 171 and cardiovascular disease (CVD) in 112 patients. Jin et al. in their retrospective epidemiological study evaluating clinical and virologic characteristics of 74 COVID-19 patients manifesting with gastrointestinal symptoms (nausea, vomiting, diarrhea) reported increased AST, but not ALT levels, which were significantly higher in patients with COVID-19 with gastrointestinal (GI) symptoms than in those without GI symptoms (29.35 vs. $24.4, p=0.02)$. Gastrointestinal symptoms were associated with more severe course of infection (in $23 \%$ of patients compared to $8 \%$ of patients without alimentary tract symptoms). Additionally, patients with gastrointestinal tract involvement had significantly higher rates of fever $>38.5^{\circ} \mathrm{C}$, fatigue, shortness of breath and headache. Based on initial univariate analysis of epidemiological, clinical and laboratory variables the authors identified 11 significant risk factors for severe/critical COVID-19, including increased operating rooms (ORs) of age $\geq 50$ years, period between illness onset and hospital visit, sputum production, any existing medical condition, multiple lung infection, ALT and lactate dehydrogenase (LDH) levels, glucose and $\mathrm{C}$ reactive protein (CRP) concentrations, as well as decreased OR of the infected area [4]. Based on these variables Jin, et al. concluded that sputum production and increased LDH/glucose levels were independent risk factors for severe/critical COVID-19 in patients with GI symptoms [4].

Most of the patients included in our systematic review were younger, below the age of 50 . Generally only ALT/AST levels were frequently reported, with scarce information on LDH, previous infections, glucose or CRP. These data were also missing in a recent meta-analysis by Parohan et al., who concluded that the incidence of liver injury may be higher in patients with severe COVID-19 infection [64]. Similarly Xu et al. [11] summarized the reports of liver injury caused by SARS-CoV, MERS-CoV and SARS-CoV-but were unable to define the mechanisms of liver injury associated with SARS-CoV-2 infection. In our systematic review the risk of severe illness could not be predicted using the variable which were reported.

\subsection{Hepatic Dysfunction Associated with ICU Procedures}

The need for organ support and intensive care treatment, regardless of the reason (i.e., acute hypoxemic respiratory failure, shock, heart failure) during the COVID-19 outbreak has proven to be substantial resulting in significant strain on health care systems around the world. Based on the available data, standard of care was observed for ICU treatment and procedures for patients with SARS-CoV-2 $[2,12,18,65]$ For sepsis diagnosis and treatment, the 2016 Third International Consensus Definition for Sepsis and Septic Shock [66] guidelines were followed; acute respiratory distress syndrome (ARDS) was diagnosed according to the Berlin Definition [67], and acute kidney injury was diagnosed according to the Kidney Disease Improving Global Outcomes (KDIGO) clinical practice guidelines [68].

Following recent guidelines, early endotracheal intubation to decrease the risk of virus aerosolization was recommended in patients with acute hypoxemic respiratory failure, including high positive end-expiratory pressure (PEEP) and prone ventilation for 12 to $16 \mathrm{~h}$ per day $[3,69]$. Hepatic dysfunction has been associated with mechanical ventilation, namely high levels of PEEP that contributes to hepatic congestion. High levels of PEEP $\left(18-20 \mathrm{~cm} \mathrm{H}_{2} \mathrm{O}\right)$, as recommended for treatment of respiratory failure associated with COVID-19 [3], may significantly increase right atrial pressure, leading to obstructed venous return from the inferior vena cava and venous congestion. However, 
according to the available data, liver blood test abnormalities were also found in patients with COVID-19 without mechanical ventilation. Based on our systematic search the data on the effect or association of mechanical ventilation and levels of PEEP and liver test anomalies are inconclusive.

As expected, non-survivors presented with higher sequential organ failure assessment (SOFA), quick sequential organ failure assessment (qSOFA) and pneumonia CURB-65 (confusion, urea, respiratory rate, blood pressure, age above 65) scores, which indicates higher disease severity and multi-organ involvement [18]. The frequency of complications was higher in non-survivors than survivors, with sepsis and ARDS being the most frequently observed complication, followed by heart failure and septic shock $[12,18]$. The mortality rates among patients requiring ICU admission ranged from $16 \%$ to $78 \%[12,18,65,70]$.

Respiratory support requirement was substantial. In studies regarding the ICU population, the reported use of non-invasive ventilation ranged from $11 \%$ [65] to $62 \%$ [70] and this value included patients receiving high-flow nasal cannula [2]. A retrospective case series by Grasselli et al. [65] involving critically ill patients with laboratory-confirmed COVID-19 admitted to ICUs in Lombardi, Italy found that $99 \%$ of patients required respiratory support, including endotracheal intubation and invasive ventilation in $88 \%$. The need for invasive mechanical ventilation in this patient population was higher than that recently reported for other ICU's ranging from $30 \%$ to $71 \%$ [12,18,20,70,71]. ExtraCorporeal Membrane Oxygenation (ECMO) therapy was used in only $0.5 \%-2 \%$ of cases $[2,12,18]$. None of the studies reported hepatic failure as the main cause of ICU admission.

\subsection{Hepatotoxicity Related to COVID-19}

Studies in SARS showed that the genetic material of the SARS-CoV-2 was present in the liver tissue, confirming direct hepatocyte infection and dysfunction. No fibrosis or fibrin deposition was found [13]. Hepatotoxicity associated with SARS-CoV-2 or any other coronavirus could therefore be a result of direct viral hepatitis or secondary to drug toxicity from antiviral drugs, steroids and/or antibiotics. The effect of an enhanced inflammatory response associated with COVID-19 cannot be ruled out. Studies reporting RNA sequencing data from intracellular viral load of two independent patient cohorts showed a significant increase of ACE2 expression in cholangiocytes (59.7\%) as compared with hepatocytes (2.6\%). This suggests an affinity of the 2019-nCoV to the bile ducts [72]. In our analysis only 12 studies reported liver histopathology, but none in SARS-CoV-2 infected patients. The descriptions included: (i) non-specific inflammation in postmortem biopsy; (ii) congestion, hemorrhage and focal perivenular loss of hepatocytes (iii) macrovesicular perivenular steatotic change, (iv) sinusoidal congestion and (v) hemorrhage or (vi) focal perivenular loss of hepatocytes. Massive hepatic necrosis was reported in only one case. Few of the studies explored the viral load in hepatic tissue, which may be relevant in light of data reporting the novel m6 A methylation loci in the S protein of SARS-CoV-2 as an underlining mechanism for its virulence and transmission capacity [4]. A recent post-mortem case study published during revisions of this review reported moderate microvesicular steatosis and mild lobular and portal activity in a patient who expired due to COVID-19, suggesting it was caused by either SARS-CoV-2 infection, but drug-induced liver injury could not be ruled out [73]. Further research regarding SARS-CoV-2 hepatotoxicity with potential new therapeutic options is necessary [7].

\subsection{Comorbidities and Liver Injury in COVID-19 Patients}

The effect of having underlying chronic liver disease remains unclear as there were a small number of subjects with chronic hepatitis B and liver cirrhosis. The association between pre-existing liver disease and SARS-CoV-2 infection needs further investigation.

Patients with type 2 diabetes mellitus (T2DM) and hypertension are not only more vulnerable to COVID-19 but also have a more serious disease course. The study by Guan et al. [2] found hypertension in $15 \%$, T2DM in $7.4 \%$ and chronic hepatitis B in $2 \%$ of patients with COVID-19. Subjects with T2DM and/or obesity which together with arterial hypertension are components of metabolic syndrome would be expected to have a higher incidence of nonalcoholic fatty liver disease (NAFLD) or nonalcoholic 
steatohepatitis (NASH) and be at higher risk for hepatic manifestations of COVID-19, but there was insufficient data to determine an association. Another study by Wang et al. [12] showed hypertension in $31 \%, \mathrm{CVD}$ in $14 \%, \mathrm{~T} 2 \mathrm{DM}$ in $14 \%$ and preexisting chronic liver disease in $3 \%$. Patients with severe COVID-19 requiring ICU care were twice as likely to have these co-morbidities. There was no particular data on NAFLD in this study. The average aminotransferases levels were within normal range for the extended cohort of patients with COVID-19, but a sub-analysis of patients with mild and severe disease revealed significantly elevated ALT and AST levels in those requiring ICU care. Total serum bilirubin concentration was also higher in these patients. The association of metabolic anomalies and higher AST and ALT levels in patients with severe course of disease suggests increased susceptibility to the hepatotoxic effect of the virus. The study by Zhou et al. [18] also described a two-fold increase of T2DM (19\% of the cohort), hypertension (30\%) and CVD (15\%) in patients with a fatal outcome. Data on preexisting liver disease and metabolic abnormalities was insufficient for study. Serum albumin concentration was significantly lower in non-survivors. In addition, ferritin and interleukin 6 (IL-6) levels alongside ALT activity increased significantly in patients with more severe disease. Both ferritin and IL-6 have been described with non-favorable course of liver injury in general [18]. Contemporaneous increase in ferritin, IL-6 and ALT levels associated with decreased albumin concentration suggest more significant liver involvement in the course of COVID-19.

Another study, by Yang et al. [20] compared 52 patients with severe COVID-19. There was higher incidence of T2DM in non-survivors. Liver dysfunction occurred with similar frequency when comparing survivors with non-survivors ( $30 \%$ vs. $28 \%$ ). However, in non-survivors, higher serum bilirubin, lower platelet count and prolonged prothrombin time were found. In the study by Shi et al. [23] 81 patients, all with pneumonia, including 7 patients with liver cirrhosis, were analyzed in respect to the severity of radiological findings on computed tomography (CT) scan. Patients with subclinical disease had significantly lower AST but not ALT activity compared to those with overt pulmonary symptoms. Liver tests including serum albumin, thrombin time, fibrinogen, platelet count and bilirubin were not different between those with and without cirrhosis, but larger studies are necessary to understand the effect of cirrhosis on COVID-19 infection and outcomes. The study by Jin et al. [4] compared patients infected with SARS-CoV-2 with and without gastrointestinal (GI) symptoms (nausea, vomiting, diarrhea). The study showed that patients with GI symptoms were more likely to experience a severe/critical disease course and had higher rates of $>38.5^{\circ} \mathrm{C}$-temperature and shortness of breath. More patients with GI symptoms had preexisting chronic liver disease (11\% vs. 3\%). Additionally, when analyzed separately, patients with chronic liver disease also had a statistically insignificant increased risk for severe/critical disease ( $23 \%$ vs. $8 \%$ ). Patients with GI symptoms had lower levels of albumin and upregulated AST activity and CRP concentration and depletion of lymphocyte count. There was no difference in ALT activity, prothrombin time and bilirubin levels when compared to patients without GI disease. These results suggest an association between the presence of GI symptoms alongside with preexisting chronic liver disease and a more severe form of COVID-19. Importantly, increased ALT activity was found to be an important identifier of severe/critical COVID-19. Additionally, patients with GI symptoms were more prone to respiratory complications and liver injury ( $6.8 \%$ vs. $2.1 \%$; $17.6 \%$ vs. $2.1 \%$, respectively). These results show that in some patients SARS-CoV-2 exerts higher affinity to the gastrointestinal tract including the liver with an increased risk for hepatic injury. Similar to SARS-CoV, SARS-CoV-2 enters the cell using ACE2 receptors [72,74] which are expressed in hepatocytes and bile duct epithelial cells (cholangiocytes). The ACE2 has also been found to be expressed on cholangiocytes [75]. Cholangiocytes play an essential role in liver regeneration and immune response [76] This suggests that the liver injury in patients with COVID-19 may result from cholangiocyte damage, and not through hepatocytes. As mentioned above, IL-6 levels were significantly upregulated especially in more severe and critical patients [18]. Normally, IL-6 has an hepatoprotective effect and adjusts hepatic regeneration. Increased levels of IL-6 derange liver regeneration, stimulate hepatocytes to produce various proinflammatory cytokines and potentiates necroinflammatory activity and liver injury $[77,78]$ Postmortem biopsies of patients with COVID-19 
disease showed moderate microvascular steatosis and mild lobular and portal inflammatory activity only. It is unclear if this is directly due to infection or a result from drug-induced liver injury.

Obesity was reported to be an additional factor of severe COVID-19 illness [79]. However, obesity status was evaluated in only two studies in our systematic search reporting only seven patients $(n=7)$ and one case report. Other reported comorbidities included asthma, COPD, kidney diseases and others with a total number of 398 patients. A total of 407 patients required mechanical ventilation; however, data were scarce and reported only in 19 studies, which limits the conclusions on potential mechanisms and role of other factors associated with liver failure in COVID-19 patients.

\subsection{SARS-CoV-1 and Liver Injury}

SARS-CoV-1 was also suggested to be associated with liver injury. Autopsies of SARS patients found large numbers of virus particles not only in the lungs but also in the hepatocytes and hepatic vascular endothelial cells [80,81]. The retrospective study by Chan et al. [24] investigated the pattern of hepatic damage as well as the effect of chronic hepatitis B on the clinical outcome of SARS. The study included $12 \mathrm{HBsAg}$-positive (including two with cirrhosis) and $106 \mathrm{HBsAg-negative} \mathrm{SARS} \mathrm{patients.}$ Among ten non-cirrhotic HBsAg-positive patients 3 were admitted for chronic hepatitis B flare-up, 5 had elevated and 2 had normal ALT levels. Among the 106 HBsAg-negative patients, 16 (15\%) patients had elevated ALT activity on admission. None of these patients had comorbid illnesses or had taken any medications. However, 71 patients with normal ALT on admission experienced an increase during hospitalization. ALT activity was not associated with severity of SARS infection and eventual complications. Overall, 66 of 80 (83\%) patients who experienced ALT elevation had normalization of ALT levels on discharge and another $10(13 \%)$ patients had decreasing levels on follow-up. Histopathology of autopsy specimens showed a lack of acute hepatitis changes or hepatic necrosis. Coronavirus particles were not detected by electron microscopy. The clinical outcomes of chronic hepatitis B patients were not different from those of HBsAg-negative patients. The absence of specific hepatic lesions in hepatitis B virus and SARS-CoV-1 coinfected patients on autopsy suggests no correlation between SARS-CoV-1 and chronic hepatitis B infection. Also, the presence of hepatitis B virus infection did not influence the clinical outcomes of SARS. The authors concluded that the liver derangement in SARS was likely a nonspecific transient reaction caused by the infection rather than by a direct cytopathic effect of SARS-CoV-1 Another study by Chan et al. [25] encompassed 294 patients, including 30 with chronic hepatitis B. Elevated ALT activity on admission was found in $24 \%$ patients, while $69 \%$ of patients developed elevated ALT during the course of illness. Twenty-eight $(9.5 \%)$ patients had ALT over $5 \times$ upper limit of normal (ULN), 7 patients had elevated serum bilirubin and 10 had abnormal prothrombin time. Forty (29\%) patients had experienced elevated ALP during the course of illness. None of the patients developed hepatic encephalopathy. Seven of the 28 (25\%) patients died of SARS and multi-organ failure. On the other hand, 5 of $89(6 \%)$ patients who had persistently normal ALT died. Among the 180 patients who had elevated ALT levels, 128 (71\%) subsequently normalized and $37(21 \%)$ patients had improved ALT level on follow-up visit. There were 28 patients who had peak ALT over $5 \times$ ULN with significant male predominance and co-morbidities. A cut-off ALT $>5 \times$ ULN was associated with oxygen desaturation OR 3.24 (95\% CI 1.23-8.59, $p=0.018)$, ICU care OR 3.70 (95\% CI 1.38-9.89, $p=0.009)$, mechanical ventilation OR $6.64(95 \%$ CI 2.22-19.81, $p=0.001)$ and death OR 7.34 (95\% CI 2.28-24.89, $p=0.001)$. Co-existing chronic hepatitis B was not associated with higher ALT level, increased risk of oxygen desaturation, ICU admission, mechanical ventilation or mortality. The authors concluded that reactive hepatitis with transiently upregulated transaminases is a common complication of SARS-CoV infection. However, contrary to the previous study, severe hepatitis indicated by higher ALT activity, had a negative impact on outcomes of SARS. The study by Yang et al. [39] with 168 patients with SARS included 17 who were HBsAg or HBeAg-positive. The authors measured variables at four time points during hospitalization (day of admission, 1st, 2nd and 3rd week after admission). Abnormal ALT activity was observed in $52.5 \%, 71.8 \%, 85.7 \%$ and $85.2 \%$ of patients, respectively, with the highest average ALT in the 4th week of hospitalization $(56.07,86.46,106.69$ and $111.32 \mathrm{U} / \mathrm{L}$, 
respectively). The average levels of serum albumin decreased during the hospitalization $(37.25,35.82$, 34.49 and $34.26 \mathrm{~g} / \mathrm{L}$, respectively). AST activity was slightly increased $(1.5 \times \mathrm{ULN})$, while total bilirubin concentration remained within normal range. Although increased, ALT activity was not related to more severe course of SARS infection defined by high fever $>39^{\circ} \mathrm{C}$, decreased blood oxygen saturation $\left(\mathrm{SaO}_{2}\right)$ and immune functional disorder. Liver biopsy in four patients did not reveal any specific findings. All patients included in the study were treated with antibiotics which could have influenced ALT activity. Chronic hepatitis B was not associated with worse liver injury. The authors concluded that liver damage of patients with SARS usually occurs in the early stage of the disease characterized by decreased albumin levels and abnormal levels of ALT. The liver damage induced by SARS seems to be primary rather than secondary due to low $\mathrm{SaO}_{2}$ or hyperthermia. Hepatotoxic drugs may play a role in increasing the severity of liver damage or prolonging the time of liver function recovery.

The association between increased ALT that occurs at the very early stage of SARS and recognition that ACE2 is an entry receptor for SARS-CoV-1 suggests that the liver is a direct target of viral infection. Liver biopsies of patients with SARS have shown a significant increase in mitotic cells, with eosinophilic bodies and ballooning hepatocytes, suggesting that SARS-CoV-1 may induce apoptosis of liver cells and thus cause liver injury [13]. Other studies showed that SARS-CoV-specific protein 7a can induce apoptosis in cell lines of different organs (including the lung, kidney and liver) through the caspase-dependent pathway, further supporting the pathogenic role of SARS-CoV-1 on hepatocytes and cholangiocytes resulting in regeneration derangement and liver injury [82].

Duan et al. [83] found ALT activity to be elevated in almost 38\% out of 154 SARS patients, with higher levels correlated with more severe disease course at the day of admission. ALT normalized in $75 \%$ out of those patients within two weeks. Serum albumin decreased in $29 \%$ of subjects. IL-1beta, IL-2, IL-4, IL-6, IL-8, IL-10, tumor necrosis factor (TNF)-alpha levels were upregulated in SARS patients within the first week of hospitalization and decreased by the fourth week. This increased cytokine expression was associated with higher ALT activity. Liver ultrasonography performed in 11 patients and histopathological examination of liver specimens in four patients did not show any characteristic features. The results suggest liver injury which occurs at the very early stage of infection to be part of a complex systemic inflammatory response syndrome (SIRS). Effective depletion of the cytokine storm may be beneficial to reduce liver impairment and improve SARS outcomes.

\subsection{MERS-CoV and Liver Injury}

MERS-CoV infection in patients is characterized by fever, cough and shortness of breath, respiratory failure in some cases and gastrointestinal syndromes affecting about $25 \%-30 \%$ of patients. MERS-CoV can cause severe infection requiring intensive care and mortality reaching $60 \%$. Most MERS patients had upregulated ALT and AST activity and hyperbilirubinemia with average increase $2 \times$ ULN, $3 \times$ ULN and $1.5 \times$ ULN, respectively. Hypoalbuminemia was another common finding in MERS patients at the day of diagnosis with frequent deterioration during the hospitalization. Concomitant infections and low albumin were independent risk factors for severe infection requiring ICU (OR $14.13,95 \%$ CI 1.58-126.09; $p=0.018$ and OR 6.31, 95\% CI 1.24-31.90; $p=0.026$, respectively) [52]. Similar observations were found in several other studies of MERS [52,84-86]. Similar to the observation in SARS patients, the pathological features of liver injury in MERS were mild portal tract and lobular lymphocytic infiltration, moderate steatosis and scattered calcifications [53]. Unlike SARS-CoV-2 and SARS-CoV-1, MERS-CoV required dipeptidyl peptidase-4 (DPP-4) as its functional receptor to enter cells and evoke infection in cells [87-89]. The lack of data makes it difficult to determine if the liver injury observed during MERS-CoV infection is the consequence of direct viral infection or is inflammation-mediated.

\section{Conclusions}

SARS-CoV-2, SARS-CoV-1 and MERS-CoV frequently affect the liver; however, numerous confounding factors could bias the interpretation of available data regarding the correlation between infection and hepatocyte damage. Pre-existing liver disease such as NAFLD and NASH or viral 
hepatitides were underreported in most the studied cohorts. Abnormal liver tests could be related to SARS, other pre-existing comorbidity treatment or other co-therapy. Liver injury which is diagnosed at the early stage of viral infection may result either from a direct insult of the virus or comprises part of the complex systemic inflammatory response syndrome associated with the infection. The mechanism of hepatic injury in SARS infections is still not elucidated, it is likely multifactorial and requires further prospective and properly designed studies. Medication used to treat COVID-19, SARS and MERS including interferon- $\alpha$ sprays, arbidol hydrochloride, lopinavir, ritonavir, antibiotics and glucocorticoids, may also account for hepatic dysfunction, but were underreported in the studied cohorts. More studies on the correlation between coronaviruses, especially SARS-CoV-2 and liver injury are necessary, including histopathology and molecular diagnostic assessments. An interaction between viral shedding of SARS-CoV-2 in stool has been reported [90], but it's association with hepatotoxicity is yet to be determined.

Supplementary Materials: The following are available online at http://www.mdpi.com/2077-0383/9/5/1420/s1, Table S1: Risk of bias assessment.

Author Contributions: Conceptualization, M.K. (Michał Kukla), K.S.-Ż. and W.M.; methodology, M.K. (Mariusz Kaczmarczyk), K.S.-Ż., D.M. and K.K.; software, M.K. (Mariusz Kaczmarczyk); validation, M.K. (Michał Kukla), K.S.-Ż., L.F.L., A.K. and W.M.; formal analysis, M.K. (Michał Kukla), K.S.-Ż., K.K., A.K. and W.M.; investigation, K.S.-Ż., D.M. and I.Ł.; resources, M.K. (Mariusz Kaczmarczyk), E.S. and W.M..; data curation, M.K. (Michał Kukla), K.S.-Ż. and D.M.; writing—original draft preparation, M.K. (Michał Kukla), K.S.-Ż., M.P.S., K.K., A.K., W.M.; writing—review and editing, M.K. (Michał Kukla), K.S.-Ż., D.M., M.P.-S., I.Ł., L.L., E.S., M.K. (Mariusz Kaczmarczyk), A.K. and W.M; visualization, K.S.-Ż.; supervision, A.K., W.M.; project administration, M.K. (Michał Kukla), W.M.; funding acquisition, A.K., W.M. All authors have read and agreed to the published version of the manuscript.

Conflicts of Interest: The authors declare no conflict of interest.

\section{References}

1. Phelan, A.L.; Katz, R.; Gostin, L.O. The Novel Coronavirus Originating in Wuhan, China: Challenges for Global Health Governance. JAMA 2020. [CrossRef] [PubMed]

2. Guan, W.; Ni, Z.; Hu, Y.; Liang, W.; Ou, C.; He, J.; Liu, L.; Shan, H.; Lei, C.; Hui, D.S.C.; et al. Clinical Characteristics of Coronavirus Disease 2019 in China. N. Engl. J. Med. 2020. [CrossRef] [PubMed]

3. World Health Organization. Clinical Management of Severe Acute Respiratory Infection (SARI) when COVID-19 Disease is Suspected: Interim Guidance. Available online: https://apps.who.int/iris/handle/10665/ 331446 (accessed on 6 April 2020).

4. Jin, X.; Lian, J.-S.; Hu, J.-H.; Gao, J.; Zheng, L.; Zhang, Y.-M.; Hao, S.-R.; Jia, H.-Y.; Cai, H.; Zhang, X.-L.; et al. Epidemiological, clinical and virological characteristics of 74 cases of coronavirus-infected disease 2019 (COVID-19) with gastrointestinal symptoms. Gut 2020. [CrossRef] [PubMed]

5. Clinical Characteristics of COVID-19 Patients with Digestive Symptoms in Hubei, China. Available online: https://www.practiceupdate.com/content/clinical-characteristics-of-covid-19-patientswith-digestive-symptoms-in-hubei-china/98000 (accessed on 6 April 2020).

6. Gu, J.; Han, B.; Wang, J. COVID-19: Gastrointestinal Manifestations and Potential Fecal-Oral Transmission. Gastroenterology 2020, 158, 1518-1519. [CrossRef] [PubMed]

7. Kotfis, K.; Skonieczna-Żydecka, K. COVID-19: Gastrointestinal symptoms and potential sources of 2019-nCoV transmission. Anaesthesiol. Intensive Ther. 2020, 52. [CrossRef]

8. Alsaad, K.O.; Hajeer, A.H.; Al Balwi, M.; Al Moaiqel, M.; Al Oudah, N.; Al Ajlan, A.; AlJohani, S.; Alsolamy, S.; Gmati, G.E.; Balkhy, H.; et al. Histopathology of Middle East respiratory syndrome coronovirus (MERS-CoV) infection-clinicopathological and ultrastructural study. Histopathology 2018, 72, 516-524. [CrossRef]

9. Zhang, Y.; Zheng, L.; Liu, L.; Zhao, M.; Xiao, J.; Zhao, Q. Liver impairment in COVID-19 patients: A retrospective analysis of 115 cases from a single center in Wuhan city, China. Liver Int. 2020. [CrossRef]

10. Bangash, M.N.; Patel, J.; Parekh, D. COVID-19 and the liver: Little cause for concern. Lancet Gastroenterol. Hepatol. 2020. [CrossRef]

11. Xu, L.; Liu, J.; Lu, M.; Yang, D.; Zheng, X. Liver injury during highly pathogenic human coronavirus infections. Liver Int. 2020. [CrossRef] 
12. Wang, D.; Hu, B.; Hu, C.; Zhu, F.; Liu, X.; Zhang, J.; Wang, B.; Xiang, H.; Cheng, Z.; Xiong, Y.; et al. Clinical Characteristics of 138 Hospitalized Patients With 2019 Novel Coronavirus-Infected Pneumonia in Wuhan, China. JAMA 2020, 323, 1061-1069. [CrossRef]

13. Chau, T.-N.; Lee, K.-C.; Yao, H.; Tsang, T.-Y.; Chow, T.-C.; Yeung, Y.-C.; Choi, K.-W.; Tso, Y.-K.; Lau, T.; Lai, S.-T.; et al. SARS-associated viral hepatitis caused by a novel coronavirus: Report of three cases. Hepatology 2004, 39, 302-310. [CrossRef] [PubMed]

14. Shamseer, L.; Moher, D.; Clarke, M.; Ghersi, D.; Liberati, A.; Petticrew, M.; Shekelle, P.; Stewart, L.A. PRISMA-P Group Preferred reporting items for systematic review and meta-analysis protocols (PRISMA-P) 2015: Elaboration and explanation. BMJ 2015, 350, g7647. [CrossRef] [PubMed]

15. Vandenbroucke, J.P.; von Elm, E.; Altman, D.G.; Gøtzsche, P.C.; Mulrow, C.D.; Pocock, S.J.; Poole, C.; Schlesselman, J.J.; Egger, M. STROBE Initiative Strengthening the Reporting of Observational Studies in Epidemiology (STROBE): Explanation and elaboration. Int. J. Surg. 2014, 12, 1500-1524. [CrossRef]

16. Chen, N.; Zhou, M.; Dong, X.; Qu, J.; Gong, F.; Han, Y.; Qiu, Y.; Wang, J.; Liu, Y.; Wei, Y.; et al. Epidemiological and clinical characteristics of 99 cases of 2019 novel coronavirus pneumonia in Wuhan, China: A descriptive study. Lancet 2020, 395, 507-513. [CrossRef]

17. Chuan, L.; Zicheng, J.; Chuxiao, S.; Hongguang, Z.; Hongmei, Y.; Zhenhuai, C.; Baoyi, M.; Weiying, L.; Huihong, H.; Jie, Y.; et al. Preliminary study of the relationship between novel coronavirus pneumonia and liver function damage: A multicenter study. Chin. J. Hepatol. 2020, 28, 148-152. [CrossRef]

18. Zhou, F.; Yu, T.; Du, R.; Fan, G.; Liu, Y.; Liu, Z.; Xiang, J.; Wang, Y.; Song, B.; Gu, X.; et al. Clinical course and risk factors for mortality of adult inpatients with COVID-19 in Wuhan, China: A retrospective cohort study. Lancet 2020. [CrossRef]

19. Zhang, C.; Shi, L.; Wang, F.-S. Liver injury in COVID-19: Management and challenges. Lancet Gastroenterol. Hepatol. 2020. [CrossRef]

20. Yang, X.; Yu, Y.; Xu, J.; Shu, H.; Xia, J.; Liu, H.; Wu, Y.; Zhang, L.; Yu, Z.; Fang, M.; et al. Clinical course and outcomes of critically ill patients with SARS-CoV-2 pneumonia in Wuhan, China: A single-centered, retrospective, observational study. Lancet Respir. Med. 2020. [CrossRef]

21. Xu, X.-W.; Wu, X.-X.; Jiang, X.-G.; Xu, K.-J.; Ying, L.-J.; Ma, C.-L.; Li, S.-B.; Wang, H.-Y.; Zhang, S.; Gao, H.-N.; et al. Clinical findings in a group of patients infected with the 2019 novel coronavirus (SARS-Cov-2) outside of Wuhan, China: Retrospective case series. BMJ 2020, 368. [CrossRef]

22. Wu, J.; Liu, J.; Zhao, X.; Liu, C.; Wang, W.; Wang, D.; Xu, W.; Zhang, C.; Yu, J.; Jiang, B.; et al. Clinical Characteristics of Imported Cases of COVID-19 in Jiangsu Province: A Multicenter Descriptive Study. Clin. Infect. Dis. 2020. [CrossRef]

23. Shi, H.; Han, X.; Jiang, N.; Cao, Y.; Alwalid, O.; Gu, J.; Fan, Y.; Zheng, C. Radiological findings from 81 patients with COVID-19 pneumonia in Wuhan, China: A descriptive study. Lancet Infect. Dis. 2020. [CrossRef]

24. Chan, H.L.Y.; Leung, W.-K.; To, K.-F.; Chan, P.K.S.; Lee, N.; Wu, A.; Tam, J.S.L.; Sung, J.J.Y. Retrospective analysis of liver function derangement in severe acute respiratory syndrome. Am. J. Med. 2004, 116, 566-567. [CrossRef] [PubMed]

25. Chan, H.-L.-Y.; Kwan, A.-C.-P.; To, K.-F.; Lai, S.-T.; Chan, P.K.-S.; Leung, W.-K.; Lee, N.; Wu, A.; Sung, J.J.-Y. Clinical significance of hepatic derangement in severe acute respiratory syndrome. World J. Gastroenterol. 2005, 11, 2148-2153. [CrossRef] [PubMed]

26. Chen, J.; Zhang, H.; Xie, Y.; Wan, J.; Lu, Z.; Wang, D.; Wang, Q.; Xue, X.; Si, W.; Luo, Y.; et al. [Morphological study of severe acute respiratory syndrome (SARS)]. Zhonghua Bing Li Xue Za Zhi 2003, 32, 516-520.

27. Cui, H.-J.; Tong, X.-L.; Li, P.; Hao, Y.-X.; Chen, X.-G.; Li, A.-G.; Zhang, Z.-Y.; Duan, J.; Zhen, M.; Zhang, B.; et al. Serum hepatic enzyme manifestations in patients with severe acute respiratory syndrome: Retrospective analysis. World J. Gastroenterol. 2004, 10, 1652-1655. [CrossRef]

28. Ding, Y.; Wang, H.; Shen, H.; Li, Z.; Geng, J.; Han, H.; Cai, J.; Li, X.; Kang, W.; Weng, D.; et al. The clinical pathology of severe acute respiratory syndrome (SARS): A report from China. J. Pathol. 2003, 200, $282-289$. [CrossRef]

29. Farcas, G.A.; Poutanen, S.M.; Mazzulli, T.; Willey, B.M.; Butany, J.; Asa, S.L.; Faure, P.; Akhavan, P.; Low, D.E.; Kain, K.C. Fatal severe acute respiratory syndrome is associated with multiorgan involvement by coronavirus. J. Infect. Dis. 2005, 191, 193-197. [CrossRef]

30. Guan, Y.; Tang, X.; Yin, C.; Yi, Z. [Study on the damage of liver in patients with SARS]. Zhongguo Wei Zhong Bing Ji Jiu Yi Xue 2004, 16, 267-270. [PubMed] 
31. Han, Y.; Geng, H.; Feng, W.; Tang, X.; Ou, A.; Lao, Y.; Xu, Y.; Lin, H.; Liu, H.; Li, Y. A follow-up study of 69 discharged SARS patients. J. Tradit. Chin. Med. 2003, 23, 214-217.

32. Hsiao, C.-H.; Wu, M.-Z.; Hsieh, S.-W.; Chien, L.-C.; Hwang, K.-C.; Su, I.-J. Clinicopathology of severe acute respiratory syndrome: An autopsy case report. J. Formos. Med. Assoc. 2004, 103, 787-792.

33. Kumar, D.; Tellier, R.; Draker, R.; Levy, G.; Humar, A. Severe Acute Respiratory Syndrome (SARS) in a liver transplant recipient and guidelines for donor SARS screening. Am. J. Transplant. 2003, 3, 977-981. [CrossRef] [PubMed]

34. Lang, Z.; Zhang, L.; Zhang, S.; Meng, X.; Li, J.; Song, C.; Sun, L.; Zhou, Y. A clinicopathological study on 3 cases of severe acute respiratory syndrome. Zhonghua Bing Li Xue Za Zhi 2003, 32, 201-204. [PubMed]

35. Liu, Z.; Li, T.; Wang, Z.; Xu, Z.; Wang, H.; Yu, Y.; Du, T.; Bai, Y.; Qiu, Z.; Lü, W.; et al. Clinical features and therapy of 106 cases of severe acute respiratory syndrome. Zhonghua Nei Ke Za Zhi 2003, 42, 373-377. [PubMed]

36. Luo, H.-T.; Wu, M.; Wang, M.-M. Case report of the first Severe Acute Respiratory Syndrome patient in China: Successful application of extracorporeal liver support MARS therapy in multiorgan failure possibly induced by Severe Acute Respiratory Syndrome. Artif. Organs 2003, 27, 847-849. [CrossRef]

37. Zhao, L.; Xing, H.; Xu, L. Effect of SARS-associated coronavirus on peripheral blood picture and liver function. Zhongguo Wei Zhong Bing Ji Jiu Yi Xue 2004, 16, 660-663.

38. Yin, C.; Wang, C.; Tang, Z.; Wen, Y.; Zhang, S.; Wang, B. Clinical analysis of multiple organ dysfunction syndrome in patients suffering from SARS. Zhongguo Wei Zhong Bing Ji Jiu Yi Xue 2004, 16, 646-650.

39. Yang, Z.; Xu, M.; Yi, J.-Q.; Jia, W.-D. Clinical characteristics and mechanism of liver damage in patients with severe acute respiratory syndrome. HBPD INT 2005, 4, 60-63.

40. Wu, K.-L.; Lu, S.-N.; Changchien, C.-S.; Chiu, K.-W.; Kuo, C.-H.; Chuah, S.-K.; Liu, J.-W.; Lin, M.-C.; Eng, H.-L.; Chen, S.-S.; et al. Sequential changes of serum aminotransferase levels in patients with severe acute respiratory syndrome. Am. J. Trop. Med. Hyg. 2004, 71, 125-128. [CrossRef]

41. Wong, W.-M.; Ho, J.C.; Ooi, G.C.; Mok, T.; Chan, J.; Hung, I.F.; Ng, W.; Lam, Y.-M.; Tam, W.-O.; Wong, B.C.Y.; et al. Temporal patterns of hepatic dysfunction and disease severity in patients with SARS. JAMA 2003, 290, 2663-2665. [CrossRef]

42. Tong, Y.; Yin, C.; Tang, X.; Jia, W. [Changes of liver function in patients with serious acute respiratory syndrome]. Zhonghua Gan Zang Bing Za Zhi 2003, 11, 418-420.

43. Shi, X.; Gong, E.; Gao, D.; Zhang, B.; Zheng, J.; Gao, Z.; Zhong, Y.; Zou, W.; Wu, B.; Fang, W.; et al. Severe acute respiratory syndrome associated coronavirus is detected in intestinal tissues of fatal cases. Am. J. Gastroenterol. 2005, 100, 169-176. [CrossRef] [PubMed]

44. Meng, Q.; Zhao, C.; Dong, P.; Hu, Z.; Hou, W.; Zhang, K.; Liang, L.; Huang, C. Clinical features of severe acute respiratory syndrome in forty-one confirmed health care workers. Zhonghua Yu Fang Yi Xue Za Zhi 2003, 37, 236-239. [PubMed]

45. Peiris, J.S.M.; Lai, S.T.; Poon, L.L.M.; Guan, Y.; Yam, L.Y.C.; Lim, W.; Nicholls, J.; Yee, W.K.S.; Yan, W.W.; Cheung, M.T.; et al. Coronavirus as a possible cause of severe acute respiratory syndrome. Lancet 2003, 361, 1319-1325. [CrossRef]

46. Al-Tawfiq, J.A.; Hinedi, K.; Abbasi, S.; Babiker, M.; Sunji, A.; Eltigani, M. Hematologic, hepatic, and renal function changes in hospitalized patients with Middle East respiratory syndrome coronavirus. Int. J. Lab. Hematol. 2017, 39, 272-278. [CrossRef]

47. Halim, A.A.; Alsayed, B.; Embarak, S.; Yaseen, T.; Dabbous, S. Clinical characteristics and outcome of ICU admitted MERS corona virus infected patients. Egypt. J. Chest Dis. Tuberc. 2016, 65, 81-87. [CrossRef]

48. Ling, Y.; Qu, R.; Luo, Y. [Clinical analysis of the first patient with imported Middle East respiratory syndrome in China]. Zhonghua Wei Zhong Bing Ji Jiu Yi Xue 2015, 27, 630-634. [CrossRef]

49. Kapoor, M.; Pringle, K.; Kumar, A.; Dearth, S.; Liu, L.; Lovchik, J.; Perez, O.; Pontones, P.; Richards, S.; Yeadon-Fagbohun, J.; et al. Clinical and Laboratory Findings of the First Imported Case of Middle East Respiratory Syndrome Coronavirus to the United States. Clin. Infect. Dis. 2014, 59, 1511-1518. [CrossRef]

50. Yousefi, M.; Dehesh, M.M.; Farokhnia, M. Epidemiological and Clinical Characteristics of Patients with Middle East Respiratory Syndrome Coronavirus in Iran in 2014. Jpn. J. Infect. Dis. 2017, 70, 115-118. [CrossRef] 
51. Sherbini, N.; Iskandrani, A.; Kharaba, A.; Khalid, G.; Abduljawad, M.; Al-Jahdali, H. Middle East respiratory syndrome coronavirus in Al-Madinah City, Saudi Arabia: Demographic, clinical and survival data. J. Epidemiol. Glob. Health 2017, 7, 29-36. [CrossRef]

52. Saad, M.; Omrani, A.S.; Baig, K.; Bahloul, A.; Elzein, F.; Matin, M.A.; Selim, M.A.A.; Mutairi, M.A.; Nakhli, D.A.; Aidaroos, A.Y.A.; et al. Clinical aspects and outcomes of 70 patients with Middle East respiratory syndrome coronavirus infection: A single-center experience in Saudi Arabia. Int. J. Infect. Dis. 2014, 29, 301-306. [CrossRef]

53. Ng, D.L.; Hosani, F.A.; Keating, M.K.; Gerber, S.I.; Jones, T.L.; Metcalfe, M.G.; Tong, S.; Tao, Y.; Alami, N.N.; Haynes, L.M.; et al. Clinicopathologic, Immunohistochemical, and Ultrastructural Findings of a Fatal Case of Middle East Respiratory Syndrome Coronavirus Infection in the United Arab Emirates, April 2014. Am. J. Pathol. 2016, 186, 652-658. [CrossRef] [PubMed]

54. Xie, H.; Zhao, J.; Lian, N.; Lin, S.; Xie, Q.; Zhuo, H. Clinical characteristics of Non-ICU hospitalized patients with coronavirus disease 2019 and liver injury: A Retrospective study. Liver Int. 2020. [CrossRef] [PubMed]

55. George Sakoulas, M.D. ACE2 Is the SARS-CoV-2 Receptor Required for Cell Entry. NEJM J. Watch 2020, 2020. [CrossRef]

56. Xu, H.; Zhong, L.; Deng, J.; Peng, J.; Dan, H.; Zeng, X.; Li, T.; Chen, Q. High expression of ACE2 receptor of 2019-nCoV on the epithelial cells of oral mucosa. Int. J. Oral Sci. 2020, 12, 8. [CrossRef] [PubMed]

57. Li, M.-Y.; Li, L.; Zhang, Y.; Wang, X.-S. Expression of the SARS-CoV-2 cell receptor gene ACE2 in a wide variety of human tissues. Infect. Dis. Poverty 2020, 9, 45. [CrossRef] [PubMed]

58. Paizis, G.; Tikellis, C.; Cooper, M.E.; Schembri, J.M.; Lew, R.A.; Smith, A.I.; Shaw, T.; Warner, F.J.; Zuilli, A.; Burrell, L.M.; et al. Chronic liver injury in rats and humans upregulates the novel enzyme angiotensin converting enzyme 2. Gut 2005, 54, 1790-1796. [CrossRef]

59. Spadoni, I.; Zagato, E.; Bertocchi, A.; Paolinelli, R.; Hot, E.; Di Sabatino, A.; Caprioli, F.; Bottiglieri, L.; Oldani, A.; Viale, G.; et al. A gut-vascular barrier controls the systemic dissemination of bacteria. Science 2015, 350, 830-834. [CrossRef]

60. Buzzetti, E.; Pinzani, M.; Tsochatzis, E.A. The multiple-hit pathogenesis of non-alcoholic fatty liver disease (NAFLD). Metab. Clin. Exp. 2016, 65, 1038-1048. [CrossRef]

61. Mouries, J.; Brescia, P.; Silvestri, A.; Spadoni, I.; Sorribas, M.; Wiest, R.; Mileti, E.; Galbiati, M.; Invernizzi, P.; Adorini, L.; et al. Microbiota-driven gut vascular barrier disruption is a prerequisite for non-alcoholic steatohepatitis development. J. Hepatol. 2019, 71, 1216-1228. [CrossRef]

62. Sorribas, M.; Jakob, M.O.; Yilmaz, B.; Li, H.; Stutz, D.; Noser, Y.; de Gottardi, A.; Moghadamrad, S.; Hassan, M.; Albillos, A.; et al. FXR modulates the gut-vascular barrier by regulating the entry sites for bacterial translocation in experimental cirrhosis. J. Hepatol. 2019, 71, 1126-1140. [CrossRef]

63. Fine, R.L.; Manfredo Vieira, S.; Gilmore, M.S.; Kriegel, M.A. Mechanisms and consequences of gut commensal translocation in chronic diseases. Gut Microbes 2020, 11, 217-230. [CrossRef]

64. Parohan, M.; Yaghoubi, S.; Seraj, A. Liver injury is associated with severe Coronavirus disease 2019 (COVID-19) infection: A systematic review and meta-analysis of retrospective studies. MedRxiv 2020. [CrossRef]

65. Grasselli, G.; Zangrillo, A.; Zanella, A.; Antonelli, M.; Cabrini, L.; Castelli, A.; Cereda, D.; Coluccello, A.; Foti, G.; Fumagalli, R.; et al. Baseline Characteristics and Outcomes of 1591 Patients Infected With SARS-CoV-2 Admitted to ICUs of the Lombardy Region, Italy. JAMA 2020. [CrossRef] [PubMed]

66. Rhodes, A.; Evans, L.E.; Alhazzani, W.; Levy, M.M.; Antonelli, M.; Ferrer, R.; Kumar, A.; Sevransky, J.E.; Sprung, C.L.; Nunnally, M.E.; et al. Surviving Sepsis Campaign: International Guidelines for Management of Sepsis and Septic Shock: 2016. Intensive Care Med. 2017, 43, 304-377. [CrossRef] [PubMed]

67. ARDS Definition Task Force; Ranieri, V.M.; Rubenfeld, G.D.; Thompson, B.T.; Ferguson, N.D.; Caldwell, E.; Fan, E.; Camporota, L.; Slutsky, A.S. Acute respiratory distress syndrome: The Berlin Definition. JAMA 2012, 307, 2526-2533. [CrossRef]

68. Acute Kidney Injury (AKI)—KDIGO. Available online: https://kdigo.org/guidelines/acute-kidney-injury/ (accessed on 7 April 2020).

69. SCCM | COVID-19 Guidelines. Available online: https://sccm.org/SurvivingSepsisCampaign/Guidelines/ COVID-19 (accessed on 7 April 2020).

70. Huang, C.; Wang, Y.; Li, X.; Ren, L.; Zhao, J.; Hu, Y.; Zhang, L.; Fan, G.; Xu, J.; Gu, X.; et al. Clinical features of patients infected with 2019 novel coronavirus in Wuhan, China. Lancet 2020, 395, 497-506. [CrossRef] 
71. Arentz, M.; Yim, E.; Klaff, L.; Lokhandwala, S.; Riedo, F.X.; Chong, M.; Lee, M. Characteristics and Outcomes of 21 Critically Ill Patients With COVID-19 in Washington State. JAMA 2020. [CrossRef]

72. Chai, X.; Hu, L.; Zhang, Y.; Han, W.; Lu, Z.; Ke, A.; Zhou, J.; Shi, G.; Fang, N.; Fan, J.; et al. Specific ACE2 Expression in Cholangiocytes May Cause Liver Damage After 2019-nCoV Infection. BioRxiv 2020. [CrossRef]

73. Xu, Z.; Shi, L.; Wang, Y.; Zhang, J.; Huang, L.; Zhang, C.; Liu, S.; Zhao, P.; Liu, H.; Zhu, L.; et al. Pathological findings of COVID-19 associated with acute respiratory distress syndrome. Lancet Respir. Med. 2020, 8 , 420-422. [CrossRef]

74. Hoffmann, M.; Kleine-Weber, H.; Krüger, N.; Müller, M.; Drosten, C.; Pöhlmann, S. The novel coronavirus 2019 (2019-nCoV) uses the SARS-coronavirus receptor ACE2 and the cellular protease TMPRSS2 for entry into target cells. BioRxiv 2020. [CrossRef]

75. Qi, F.; Qian, S.; Zhang, S.; Zhang, Z. Single cell RNA sequencing of 13 human tissues identify cell types and receptors of human coronaviruses. Biochem. Biophys. Res. Commun. 2020, 526, 135-140. [CrossRef]

76. Banales, J.M.; Huebert, R.C.; Karlsen, T.; Strazzabosco, M.; LaRusso, N.F.; Gores, G.J. Cholangiocyte pathobiology. Nat. Rev. Gastroenterol. Hepatol. 2019, 16, 269-281. [CrossRef]

77. Selzner, N.; Selzner, M.; Tian, Y.; Kadry, Z.; Clavien, P.-A. Cold ischemia decreases liver regeneration after partial liver transplantation in the rat: A TNF-alpha/IL-6-dependent mechanism. Hepatology 2002, 36, 812-818. [CrossRef]

78. Kukla, M.; Mazur, W.; Bułdak, R.J.; Zwirska-Korczala, K. Potential role of leptin, adiponectin and three novel adipokines-visfatin, chemerin and vaspin-in chronic hepatitis. Mol. Med. 2011, 17, 1397-1410. [CrossRef] [PubMed]

79. Dietz, W.; Santos-Burgoa, C. Obesity and its Implications for COVID-19 Mortality. Obesity 2020. [CrossRef] [PubMed]

80. Ding, Y.; He, L.; Zhang, Q.; Huang, Z.; Che, X.; Hou, J.; Wang, H.; Shen, H.; Qiu, L.; Li, Z.; et al. Organ distribution of severe acute respiratory syndrome (SARS) associated coronavirus (SARS-CoV) in SARS patients: Implications for pathogenesis and virus transmission pathways. J. Pathol. 2004, 203, 622-630. [CrossRef] [PubMed]

81. Li, W.; Moore, M.J.; Vasilieva, N.; Sui, J.; Wong, S.K.; Berne, M.A.; Somasundaran, M.; Sullivan, J.L.; Luzuriaga, K.; Greenough, T.C.; et al. Angiotensin-converting enzyme 2 is a functional receptor for the SARS coronavirus. Nature 2003, 426, 450-454. [CrossRef]

82. Tan, Y.-J.; Fielding, B.C.; Goh, P.-Y.; Shen, S.; Tan, T.H.P.; Lim, S.G.; Hong, W. Overexpression of 7a, a protein specifically encoded by the severe acute respiratory syndrome coronavirus, induces apoptosis via a caspase-dependent pathway. J. Virol. 2004, 78, 14043-14047. [CrossRef]

83. Duan, Z.; Chen, Y.; Zhang, J.; Zhao, J.; Lang, Z.; Meng, F.; Bao, X. Clinical characteristics and mechanism of liver injury in patients with severe acute respiratory syndrome. Zhonghua Gan Zang Bing Za Zhi 2003, 11, 493-496.

84. Hwang, S.-M.; Na, B.-J.; Jung, Y.; Lim, H.-S.; Seo, J.-E.; Park, S.-A.; Cho, Y.-S.; Song, E.-H.; Seo, J.-Y.; Kim, S.-R.; et al. Clinical and Laboratory Findings of Middle East Respiratory Syndrome Coronavirus Infection. Jpn. J. Infect. Dis. 2019, 72, 160-167. [CrossRef]

85. Arabi, Y.M.; Al-Omari, A.; Mandourah, Y.; Al-Hameed, F.; Sindi, A.A.; Alraddadi, B.; Shalhoub, S.; Almotairi, A.; Al Khatib, K.; Abdulmomen, A.; et al. Critically Ill Patients with the Middle East Respiratory Syndrome: A Multicenter Retrospective Cohort Study. Crit. Care Med. 2017, 45, 1683-1695. [CrossRef]

86. Assiri, A.; Al-Tawfiq, J.A.; Al-Rabeeah, A.A.; Al-Rabiah, F.A.; Al-Hajjar, S.; Al-Barrak, A.; Flemban, H.; Al-Nassir, W.N.; Balkhy, H.H.; Al-Hakeem, R.F.; et al. Epidemiological, demographic, and clinical characteristics of 47 cases of Middle East respiratory syndrome coronavirus disease from Saudi Arabia: A descriptive study. Lancet Infect. Dis. 2013, 13, 752-761. [CrossRef]

87. Raj, V.S.; Mou, H.; Smits, S.L.; Dekkers, D.H.W.; Müller, M.A.; Dijkman, R.; Muth, D.; Demmers, J.A.A.; Zaki, A.; Fouchier, R.A.M.; et al. Dipeptidyl peptidase 4 is a functional receptor for the emerging human coronavirus-EMC. Nature 2013, 495, 251-254. [CrossRef] [PubMed]

88. Boonacker, E.; Van Noorden, C.J.F. The multifunctional or moonlighting protein CD26/DPPIV. Eur. J. Cell Biol. 2003, 82, 53-73. [CrossRef] [PubMed] 
89. Multi-Organ Damage in Human Dipeptidyl Peptidase 4 Transgenic Mice Infected with Middle East Respiratory Syndrome-Coronavirus. Available online: https://journals.plos.org/plosone/article?id=10.1371/ journal.pone.0145561 (accessed on 9 April 2020).

90. Cheung, K.S.; Hung, I.F.; Chan, P.P.; Lung, K.C.; Tso, E.; Liu, R.; Ng, Y.Y.; Chu, M.Y.; Chung, T.W.; Tam, A.R.; et al. Gastrointestinal Manifestations of SARS-CoV-2 Infection and Virus Load in Fecal Samples from the Hong Kong Cohort and Systematic Review and Meta-analysis. Gastroenterology 2020. [CrossRef] [PubMed]

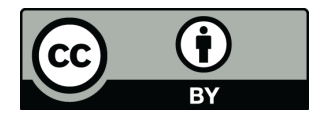

(C) 2020 by the authors. Licensee MDPI, Basel, Switzerland. This article is an open access article distributed under the terms and conditions of the Creative Commons Attribution (CC BY) license (http://creativecommons.org/licenses/by/4.0/). 